Les sources de l'Histoire des Mines : Nouveaux outils, Nouvelles approches

\title{
Mémoire sur la construction des vaisseaux dans lequel il y a une méthode pour en conduire les façons
}

Retranscription critique

Jean-Jacques Brioist et Hélène Vérin

\section{OpenEdition}

\section{Journals}

Édition électronique

URL : http://journals.openedition.org/dht/1229

DOI : $10.4000 /$ dht. 1229

ISSN : 1775-4194

\section{Éditeur :}

Centre d'histoire des techniques et de l'environnement du Cnam (CDHTE-Cnam), Société des élèves du CDHTE-Cnam

\section{Édition imprimée}

Date de publication : 1 décembre 2008

Pagination : 143-168

ISBN : 978-2-95-30779-2-6

ISSN : 0417-8726

Référence électronique

Jean-Jacques Brioist et Hélène Vérin, « Mémoire sur la construction des vaisseaux dans lequel il y a une méthode pour en conduire les façons », Documents pour l'histoire des techniques [En ligne], 16 | $2^{\mathrm{e}}$ semestre 2008, mis en ligne le 29 décembre 2010, consulté le 08 septembre 2020. URL : http:// journals.openedition.org/dht/1229; DOI : https://doi.org/10.4000/dht.1229 


\title{
Mémoire sur la construction des vaisseaux dans lequel il y a une méthode pour en conduire les façons
}

\section{( retranscription critique )}

\author{
Jean-Jacques Brioist \\ Service de la Navigation du Nord \\ Hélène Vérin \\ CNRS Centre A. Koyré
}

[f॰5] II n'y a rien de plus negligé dans la marine de la part des navigateurs, que la construction des vaisseaux, ceux qui ont servi à la mer jusqu'à ces derniers temps ne s'estant pas imaginé qu'il y a quelque raport entre la façon' d'un vaisseau et la partie de la navigation qu'on appelle la manœuvre'2, ce n'est

1 Selon l'article 'Façons d'un vaisseau' de l'Encyclopédie, ou dictionnaire raisonné des sciences, des arts et des métiers de Diderot et d'Alembert, Paris, Briasson, David l'Aîné, Lebreton, Durand imprimeurs, 1751, « On entend par ce mot, cette diminution qu'on fait à l'avant et à l'arrière du dessous du vaisseau ». Pour définir le terme, Eric Rieth cite Duranti de Lironcourt, Instruction élémentaire et raisonnée sur la construction pratique des vaisseaux, en forme de dictionnaire. Paris, J.B.G. Musier fils, 1771, p. 121. « la carène ou la partie submergée des vaisseaux diminue de capacité en arrière et en avant, dans le rapport qui, suivant l'espèce de vaisseau, convient à chacune de ses extrémités, afin qu'elles aient les propriétés et les qualités qu'on doit en attendre. Cette diminution des capacités à l'avant et à l'arrière " s' opère non seulement par le rétrécissement de la largeur du vaisseau dans ces parties, mais encore par l'acculement des varangues ou fourcats de ces couples (soit dans la dimension verticale). C'est la réunion de ces deux ouvrages qu'on nomme les façons, et c'est de leur accord que dépendent plusieurs qualités essentielles du vaisseau " Eric Rieth, Le maître-gabarit la tablette et le trébuchet,Paris, edº du CTHS, 1996, p.44.

2 « manœuvre, en terme de marine désigne tout mouvement, opération, qui nécessite un changement d'allure ou de direction dans le cap ; tels sont l'action de gouverner, les virements de bord, l'appareillage ou le movillage ". Emile Littré Dictionnaire de la langue française, 1873. Les définitions des termes de marine du Littré sont tirées du Glossaire nautique de Augustin Jal, Paris, Firmin Didot, 1848.

D'après l'article "Manœuvre " de l'Encyclopédie: "Mrs. les chevaliers de Tourville, du Guay-Trouin, Bart, du Quesne poussèrent la pratique de la manœuvre à un point de perfection, dont on ne l'auroit pas cru susceptible. Leur capacité dans cette partie de l'art de naviguer, n'étoit cependant fondée que sur beaucoup de pratique \& une grande connoissance de la mer. À force de tâtonnement, ces habiles marins s'étoient fait une routine, une pratique de manœuvrer d'autant plus surprenante, qu'ils ne la devoient qu'à leur génie. Nulle règle, nul principe proprement dit ne les dirigeoit, \& la manœuvre n'étoit rien moins qu'un art ». Encyclopédie, ou dictionnaire raisonné des sciences, des arts et des métiers. C'est néammoins comme un « art de soumettre le mouvement des vaisseaux à des lois, pour les diriger le plus avantageusement qu'il est possible » que Belin, auteur de l'article, définit le terme de 'manœuvre'.

Dans son Histoire des mathématiques, Montucla, après avoir remarqué que " quoique la manœuvre ou l'art de conduire un vaisseau au moyen des puissances mécaniques du vent, de la voile ou de la rame, soit une des parties les plus essentielles de la navigation, ce n'est que bien tard que les mathématiciens en ont fait l'objet de leurs spéculations (...) 


\section{Mémoire sur la construction des vaisseaux}

pas qu'ils n'ayent veu en gros qu'on pouvoit tirer de grands avantages d'un vaisseau qui avoit esté heureusement construit, mais ils n'ont point veu selon toutes les apparances qu'il y en avoit beaucoup à tirer de la connoissance de sa façon dans la manœuvre, et des effets remarqués par la Manœuvre à la mer ${ }^{3}$, dans la maniere de construire dans les ports ${ }^{4}$, et tout au contraire, la

je ne vois personne avant le chevalier Renau, né en 1652, qui ait considéré ce sujet avec l'attention qu'il méritoit. ॥ Notons qu'il précise plus loin : « parmi les puissances qui servent à la manœuvre d'un vaisseau, il n'en est point de plus importante que celle du gouvernail. Car, à quoi serviroient les voiles et toutes les puissances qui peuvent pousser un vaisseau en avant, s'il n'avoit la faculté de se diriger à volonté, et cette faculté dépend du gouvernail qui lui fait faire tous les mouvemens et les évolutions qu'on juge à propos », Jean Étienne Montucla, Histoire des mathématiques, dans laquelle on rend compte de leurs progrès depuis leur origine jusqu'à nos jours; où l'on expose le tableau \& le développement des principales découvertes, les contestations qu'elles ont fait naître, \& les principaux traits de la vie des mathématiciens les plus célebres, Nouvelle édition considérablement augmentée..., Paris, Henri Agasse, an VII-an X (1799-1802) volume IV.(1 ère édition 1758), p. 410 et 429 reproduction et réédition, Paris, A. Blanchard, 1960 et 1968.

3 C'est ce que remarquait Duranti de Lironcourt (cf note 1). Pour donner quelques exemples, un vaisseau étroit sera un vaisseau de près c'est-à-dire qu'il fera un bon cap contre le vent; un vaisseau large sera meilleur au portant c'est à dire à l'allure du vent arrière ; avec un fort tirant d'eau il sera peu sensible à la gîte (inclinaison du bateau sur le côté sous la pression du vent sur les voiles) et plus un vaisseau restera plat sur l'eau plus il ira vite et plus il répondra au gouvernail.

4 Par cette déclaration Renau entre dans un sujet depuis longtemps débattu, et qui fit l'objet de tout un ensemble d'enquêtes, de mesures, de règlements dans lesquels s'inscrit la proposition qu'il fait dans ce mémoire. Les rapports "entre la façon d'un vaisseau, et la partie de la navigation qu'on appelle la manœuvre " sont bien connus, en général, des navigateurs. Pour prendre un exemple bien antérieur, Fernando Oliveira dans son Livro da fabrica das naus daté des années 1580, étudiant la construction d'un modèle de navire, remarque que

«L'élévation et l'étroitesse des fonds (...) sont dictées par des hommes entendus dans l'art de la navigation, de telle sorte qu'ils donnent au bateau une forme ovale : qu'ils considèrent propres à la bonne navigation : et ceci confirme ce que j'ai dit auparavant quand je parlais de la prove. Celle-ci doit avoir une forme qui n'est ni émoussée ni tranchante, plutôt comme celle d'un œuf : car une telle forme divise les eaux et les laissent, comme je l'ai dit ».

« Alevantar o fondo, \& recolhelo (...), ordenarão os homēs entendidos na arte da navegação, a fĩ de formar os navios em figura oval ; a qual acharão ser apta pera bem navegar : por que he cõforme as que a bayxo direy, quando falar da proa. A qual ha de ser de tal forma, que nem seja de todo romba, nem aguda, como são os ouos : por que esta tal forma abre as aoguas, e espedese dellas, da maneyra que laa direy. „ Fernando Oliveira, O Livro da fabrica das naus, Lisbonne, Academia de marinha, 1991, p. 95.

Juger des rapports entre construction et manœuvre faisait partie des obligations de service des officiers de mer. L'une des consignes essentielles de Colbert à cet égard, concernait l'observation des règles à respecter au cours du désarmement des vaisseaux après une campagne. L'intendant du port devait « faire un rapport exact à chaque capitaine de la navigation de son vaisseau, contenant les observations qu'il aura pu faire en le gouvernant et le manœuvrant, les défauts qu'il aura reconnus dans sa construction, s'il ne porte pas bien ses voiles, s'il est trop pesant, si cela tient à sa masture ou aux envergures... ॥. Ce rapport devait être signé de l'intendant, du commandant du port et du capitaine du vaisseau, qui engageaient ainsi leur responsabilité. Ensuite de quoi il était envoyé au roi qui, après délibération du conseil de marine, faisait parvenir au port « ses ordres nécessaires sur ce sujet ». Les ordres concernaient en particulier les défauts qui auraient pu être remarqués dans la construction. Dans ce cas, le roi renvoyait aux maîtres-charpentiers concernés, un 
plus part de ceux qui naviguent aujourd'huy, la renferment dans leur devoir, et croyent estre en droit de decider de tous les points de la construction; par des effets qu'ils ont assez souvent mal observez, et qui ont d'autres causes que celles qu'ils jugent, puisqu'on prouveroit quelque fois sans beaucoup de peine, que celles qu'ils citent produiroient des effets tous contraires ${ }^{5}$. De tous ces gens là, il y en a peu qui touchent à une partie de la construction qu'on peut appeller la methode de conduire les façons, à cause qu'il n'est pas sy facile d'en discourir parce qu'il faudroit expliquer certaines proprietes de lignes courbes ${ }^{6}$, pour en

avis « afin qu'ils s'en corrigent à l'avenir et deviennent plus habiles que ceux des autres nations estrangeres pour la force des vaisseaux, leur bonne assiette et leur vitesse ". On voit que c'est tout un système d'évaluation et de décision qui avait été mis en place, où intervenaient et s'engageaient des compétences diverses. Voir par exemple « Instruction pour le Sieur Matharel, conseiller du Roy en ses conseils, que Sa Majesté envoye au port de Toulon pour faire les fonctions d'Intendant ॥, P. Clément, Lettres de Colbert..., Paris, 1868, †. III, 1, p. 228.

5 Renau exprime ici l'opinion du roi et de Seignelay. On peut rappeler par exemple qu'Arnoul, intendant à Toulon, ayant écrit au roi en septembre 1678 que, sur le sujet de la façon des vaisseaux, « les observations de Mr Duquesne (...) estoient tres bonnes et qu'il ne fallait rien laisser à la discretion des charpentiers », le roi opposa fermement que « cette decision est tres dangereuse, les meilleurs officiers pour la manœuvre et le combat n'ayant souvent que des connoissances tres bornées sur l'art de la construction qui demande une etude de geometrie que peu d'officiers ont esté en estat de faire ». Et il ajoute : « Mr Dugué (Trouin) le meilleur manœuvrier qui soit, estoit dans ce cas et raisonnoit pitoyablement sur ces matieres dont il vouloit pourtant decider. II faut consulter l'experience des officiers, mais raisonner beaucoup sur les faits qu'ils rapportent pour ne pas donner trop de creance aux motifs qu'ils alleguent et où ils se meprennent souvent (...) le mieux est de faire naviguer beaucoup les constructeurs mesmes ॥. BnF n.a.f. ms 9481, fo 181, 7 septembre 1678, Memoire du Roy à M. Arnoul.

6 Ainsi que le montre la suite du mémoire, c'est sur la capacité à décrire et mesurer des arcs de courbe plus généraux que des arcs de cercle que Renau juge du savoir-faire de celui qui conduit les façons de vaisseau.

II faut préciser que les maîtres charpentiers les plus avertis n'utilisent depuis le XVIe siècle jusqu'au début du XVIIIle siècle que des courbes constituées d'arcs de cercle, comme l'anse de panier à trois ou cinq centres. Dans son Livre de construction des vaisseaux (1683), François Coulomb qui enseigne à l'école de la construction de Toulon, montre l'utilisation des arcs de cercle pour dessiner certaines pièces (BnF n.a.f. 4670).

Pour saisir ce qui est en jeu dans cette remarque de Renau il faut rappeler quelques uns des principes de construction des vaisseaux mis en œuvre par les maîtres-charpentiers. Leurs méthodes qui se transmettent de père en fils sont différentes, mais toutes appartiennent au même principe de construction à franc-bord, « membrure première » qui a pris le pas sur toute autre depuis le XVe siècle. Par « membrure première ॥ il faut entendre que la forme générale de la carène est conduite en s'appuyant sur les contours des membrures (les couples transversaux) principales et surtout et avant tout le maître-couple qui définit la plus grande largeur avec, vers l'avant et vers l'arrière, des « couples de balancement ". Maître-couple et couples de balancement sont tracés à l'aide d'arcs de cercle, les couples intermédiaires étant façonnés par modifications successives de la figure du maîtrecouple, à l'aide d'instruments comme la tablette et le trébuchet. C'est en appliquant des lisses, longues planches souples, sur ces couples, que les maîtres-charpentiers formaient la carène. À partir de là, plusieurs méthodes existaient dont certaines ont été décrites dans les traités du XVIII ${ }^{e}$ siècle. Elles se distinguent par le nombre de couples façonnés et mis en place avant les lisses. On peut faire deux remarques à partir de ces quelques indications : La première est que l'on considère comme un progrès le fait de réduire le recours aux lisses, et donc à une évaluation des formes "à l'œil » au profit d'une multiplication des couples de levée dont les contours sont rigoureusement proportionnés les uns par rapport 


\section{Mémoire sur la construction des vaisseaux}

parler profondement et certaines definitions de geometrie qu'ils Ignorent pour raisonner de la manière qu'ils font presque de toutes les autres choses de la mesme nature ${ }^{7}$. Les charpentiers mesmes n'ont peu satisfaire [ [5 $\left.\mathbf{v}^{\circ}\right]$ jusqu'icy là dessus faute de ne s'estre portes à autre chose qu'à leur pratique grossiere, et fautive, pour n'avoir part en aucun principe qui les y peust mener ${ }^{8}$; ll y a ce

aux autres. Dans cette logique-là il est clair que la proposition de Renau de contrôler géométriquement les contours des couples de levée va dans le sens d'un progrès.

La deuxième remarque concerne ce que Renau évoque par « certaines propriétés des lignes courbes " ce qui renvoie, dans la pratique, à la construction géométrique du maître couple et des couples de balancement qui se faisait à l'aide d'arcs de cercles. A cet égard, E. Rieth signale que "L'usage du maître-gabarit, de la tablette et du trébuchet permet la modification de l'ensemble de la figure du maître-couple jusqu'aux couples de balancement, dans la mesure où le tracé de ces trois sections de référence repose sur une construction géométrique (plusieurs arcs de cercle) identique. Sans doute est-ce à ce niveau que se manifeste l'une des différences majeures de conception entre la méthode en usage dans les ports de France, décrite par La Madeleine ou Duhamel du Monceau au XVIIIle siècle, et celle des constructeurs anglais. Ainsi, selon Matthew Baker, I'un des plus célèbres constructeurs anglais du XVle siècle, le maître-couple d'une part, et chacune des deux sections de référence avant et arrière (plus ou moins assimilables à des couples de balancement) d'autre part, sont définies à partir d'une construction géométrique différente (quatre arcs de cercle tangents pour le maître-couple, trois pour les sections de référence avant et arrière). Seul l'arc de cercle déterminant le tracé des varangues est identique dans les trois sections. De ce fait la méthode du maître-gabarit, de la tablette et du trébuchet qui s'applique à la totalité de la figure du couple ne peut pas être employée dans la méthode de conception anglaise sauf pour la modification du tracé de la varangue ॥. E. Rieth, Le maître-gabarit, la tablette et le trébuchet, Paris, éd. du CTHS, 1996, p. 78.

On peut remarquer enfin que Renau a eu des prédécesseurs dans l'emploi de courbes algébriques non circulaires pour former les gabarits : Matthew Baker (voir M. Daeffler Formes de Carène et Navires de Combat: L'invention du Vaisseau de Ligne en Angleterre (15601642), Caen, 2004) et sans doute aussi T. Harriot (voir Jon V. Pepper, « Harriot's manuscript on shipbuilding and rigging », Proc. 3rd Int. Reunion Hist. Naut. Sc. and Hydr. "Five Hundred Years of Nautical Science ", Greenwich 1979).

7 Comme on le voit ici, Renau reprend les mêmes idées, et presque les mêmes termes utilisés dans le passage du Mémoire du Roi à Arnoul cité dans la note 2. Renau a pu contribuer à la rédaction de ce mémoire, et en tous cas, sa position est dans le droit fil de celle des Colbert. L'empirisme de principe des officiers de mer s'appuyait sur un mépris aristocratique pour la science. Elle se manifesta à de nombreuses reprises du temps de Renau, par le refus des aspirants à suivre les cours de construction organisés par Colbert dans les écoles des gardes de la marine des différents ports de guerre. Toutes sortes de sanctions et de récompenses furent envisagées pour les y contraindre. Autre manifestation de ce rejet de la science, selon Pierre Bouguer, la joute étrange qui opposa Tourville, défenseur des pratiques empiriques et le père Paul Hoste, professeur d'hydrographie à l'école des gardes de Toulon de 1686 à 1700 et auteur d'un Traité de la construction des vaisseaux (Lyon 1697): chacun fit construire un modèle de navire, celui-ci, selon la tradition des charpentiers, celvi-là, selon les principes mathématiques qu'il avait cru pouvoir appliquer. Hoste échoua lamentablement, à la grande joie des officiers de marine. Pierre Bouguer, dans la préface de son Traité du navire (1756) déplore les tristes effets de ce triomphe d'un Tourville glorieux à peu de frais, sur les progrès de la construction navale. Au XVIIIle siècle, de nombreux officiers contribuèrent activement au perfectionnement de la construction navale en s'appliquant à décrire précisément les méthodes des charpentiers, comme l'officier de vaisseaux La Madeleine (voir E. Rieth, op. cit.) , mais également, au fur et à mesure que la physique du navire progressait, en contribuant à la rendre opératoire.

8 Comme nous l'indiquions dans la note 6, pour régler les façons à partir des couples,

146 Documents pour l'histoire des techniques $\mathrm{n}^{\circ} 16$ - décembre 2008 
pendant quelque personnes qui croyant sçavoir toute L'estendüe de la geometrie, parce qu'ils sçavent tirer quelque perpendiculaire, faire quelque division de lignes, enfin resoudre quelque problesme de geometrie pratique par l'usage du compas et de la regle, s'imaginent sçavoir autant qu'il se peut sur cette partie de la construction, tout ce qu'on peut dire là dessus en général, c'est qu'il est necessaire de beaucoup de geometrie particulierement de la mechanique et de beaucoup de pratique pour parvenir à quelque chose de methodique et de certain' ; pour faire veoir cela avec quelque sorte d'evidence, il ny a qu'à expliquer une question, que Mr. Deanne ${ }^{10}$ me dit que le Roy avoit fait à Versailles, allant veoir les yackes quil amena d'Angletaire pour sa Majesté ${ }^{11}$; pourquoy un

les charpentiers disposaient d'une géométrie instrumentale qui, avec la règle et les compas, recourait à des gabarits, des tables de proportions, des tablettes, et des trébuchets. L'usage combiné de ces instruments permettait tout à la fois de contrôler les formes produites selon des proportions toujours identiques et de déterminer les grandeurs selon les conditions particulières, propres à chaque construction : tant en matériaux disponibles que modèle et dimensions exigées. Chaque maître recourait à des astuces particulières qui se transmettaient dans la famille et constituaient proprement le secret de métier. Cette géométrie instrumentale est de celles que décrivent les mécaniciens de l'antiquité et que I'on retrouve dans le livre $X$ du De Architectura de Vitruve pour la fabrique des instruments et engins de guerre, où toutes les dimensions se règlent sur un module initial selon des proportions préétablies.

Notons que si, dans l'architecture civile et militaire, l'obligation de réaliser des plans et profils des bâtiments existait depuis François ler, ce n'est qu'en 1683 qu'une ordonnance du roi obligea les maîtres-charpentiers à dresser, outre « un modèle en carton, un plan ou coupe perpendiculaire avec une coupe horizontale de chaque vaisseau, lesquels modèles et plans seront mis en dépôt dans les mains du contrôleur de la marine en chaque port pour y avoir recours lorsqu'elle voudra en faire bastir de pareils " (16 septembre 1683, CHAN Mar B2 $\left.48 \mathrm{f}^{\circ} 340 v^{\circ}\right)$. cette pratique était étrangère à leur méthode; on fit donc appel à des architectes pour ce faire. C'est le cas d'un certain Chaumont, architecte parisien, qui dessina des plans de vaisseaux construits à Toulon.

9 C'est en portant la question, non pas sur les rapports entre construction et manoeuvre, mais sur les rapports entre méthodes de construction et méthodes de manoeuvre - toutes deux appuyées sur des démonstrations géométriques - que Renau espère surmonter I'hétérogénéité des considérations sur le navire.On peut remarquer au passage le ton très cartésien de sa déclaration.

10 Antony Deane (1638-1721), célèbre constructeur anglais, membre de l'administration de la marine, avait acquis une position sociale prestigieuse (il obtint le titre de Chevalier en 1673) lorsqu'il vint à Versailles. En 1670, il écrivit un traité d'architecture navale, sur les instances de Samuel Pepys, publié par Brian Lavery, Deane's doctrine of Naval Architecture, 1670, Conway Maritim Press, 1981. La méthode qu'il expose avec un grand souci pédagogique est toute géométrique et ne se préoccupe pas de considérations de physique du navire. Elle donne seulement les moyens graphiques de reproduire précisément des formes selon des proportions et des dimensions préétablies.

11 En 1674, le roi avait commandé à Antony Deane la construction de deux jacks pour sa flotte du grand canal de Versailles. Ils devaient avoir 49 pieds de long et 13 1/2 de large (environ $16 \mathrm{~m}$ sur $4 \mathrm{~m}$ 60). La correspondance échangée entre Colbert et Henri de Massue Ruvigny, diplomate auprès de l'ambassadeur de France à Londres, Colbert de Croissy, permet de suivre les péripéties de leur construction et en particulier de leur décoration. Le roi « ne pouvant se contenter ny du dessein ny mesme des ornemens que les anglois pourroient faire ", le peintre du roi Le Brun en fut chargé. Les premiers dessins qu'il fit parvenir à l'ambassade à Londres ayant été brûlés par erreur, il fallut en faire un deuxième envoi, que les sculpteurs et peintres français arrivés à Portsmouth durent attendre, ce qui retarda d'autant l'achèvement des jacks. Ceux-ci entrèrent dans le port du Havre le 9 août 1675 à minuit. Le 13 ils arrivaient à Roven et remontèrent la Seine jusqu'à St Cloud. Les « machines » 


\section{Mémoire sur la construction des vaisseaux}

vaisseau va pour ainsy dire contre le vent, c'est à dire d'un vent de Nord, à l'est nord'est ou à l'ouest nord'ouest, et sur ce que je luy dit et à M. Desclouzeau'2, qu'on pouvoit non seulement rendre raison de cela, mais encore determiner l'endroit où le vaisseau doit aller par toutes ses voilles, certaines choses estant supposees ${ }^{13}$, il m'obligea à en faire une demonstration, que je mettray icy, parce qu'aussi bien elle me servira à establir la methode que je pretends donner par ce memoire pour conduire les façons des vaisseaux par beaucoup de raisons de constructions qui y sont enfermees; mais comme j'ay besoin pour cela de quelques propositions du mouvement loccal ${ }^{14}$, je commenceray par les expliquer pour venir ensuite $a(u)$ vaisseau ;

On appelle determination ${ }^{15}$, ce qui fait qu'une chose qui se meut [ [ ${ }^{\circ}$ 6] va plustost d'un costé que d'un autre, ainsy la disposition que cette chose a à se mouvoir

et les hommes qui devaient assurer leur transport de St Cloud à Versailles furent emmenés de Portsmouth. Parti du Havre le 22 septembre, Deane se rendit à Versailles en carrosse, accompagné de son fils, d'un interprète et de Desclouzeaux, commissaire général de la marine au Havre. La scène que décrit Renau peut être placée à la fin du mois d'août ou au début de septembre 1675.

Dans la correspondance qu'il adresse à Vauvré, intendant au Havre et au duc de $\mathrm{S}^{\dagger}$ Aignan, commandant de la place, Colbert multiplie les recommandations de traiter A. Deane avec la plus grande considération, rappelant que « ledit Sr Deane est intendant de toute la marine d'Angleterre et que son maître l'a fait chevalier depuis peu de temps ॥. Cette promotion sociale d'un constructeur de vaisseaux n'est alors pas envisageable en France. Deane comme les équipages qui accompagnèrent les jacks furent traités royalement. AN Mar B2 30, 31, B3 19 et B7 474.

12 Note en marge du manuscrit de la Bnf : « Hubert Champy, Desclouzeaux Commissaire général au havre de 1676 à 1680, à Dunkerque en 1680, Intendant à Brest 12 décembre 1683, Commissaire général à Rochefort 1675 ॥.

13 Dans le compte rendu que fait Renau de cette conversation, il semble que Deane et Desclouzeaux n'aient pas été capables de répondre à la question du roi, n'étant pas à même de " rendre raison » de cette manœuvre ni d'en " rendre compte " précisément. Avec cette référence à " certaines choses étant supposées », Renau signale qu'il entre dans le discours scientifique.

14 L'expression « mouvement local » appartient à la philosophie naturelle. Aristote réserve le terme de « mouvement » à l'évolution par degrés graduels vers un autre état, sans aucune idée spatiale. II distingue ensuite trois modalités de mouvement : le mouvement selon la qualité (chaud/froid), selon la quantité (augmentation/diminution), et enfin le mouvement local, c'est-à-dire le changement de lieu ou de position, que nous appelons aujourd'hui " déplacement " en mécanique (Physique, Livre III). La scolastique médiévale (Buridan, Bradwardine, Suiseth, Oresme) faisait un usage courant de cette terminologie aristotélicienne, qui est encore celle de Tartaglia, puis de Galilée. Quant à Descartes, sa théorie est que tout mouvement - tout changement dans l'univers - est réductible à une question de mouvement local, c'est-à-dire de mécanique. Notons aussi qu'au début de la préface de la Statique (1673), le physicien Pardies déclare : « ce traitté est une suite d'un discours sur le Mouvement local, qu'on avoit déjà publié, dans le dessein de faire une mécanique entière et de réduire en ordre toute la science du mouvement ॥. De même, Cordemoy, disciple de Descartes, emploie l'expression comme la plupart des contemporains. Voir Paul Mouy, Le développement de la physique cartésienne, Paris, Vrin, 1934, p. 101.

15 Le concept de détermination d'un mobile participe d'une conception du mouvement très dépaysante pour nous. II a été introduit par Descartes, d'abord dans son Traité du Monde posthume (rédigé en 1632, publié en 1661), puis au Discours II de la Dioptrique de 1637. Cette " détermination ॥ correspond pour l'essentiel, en termes modernes, à la direction du vecteur vitesse du mobile, sans aucune valeur d'intensité : Descartes montre 

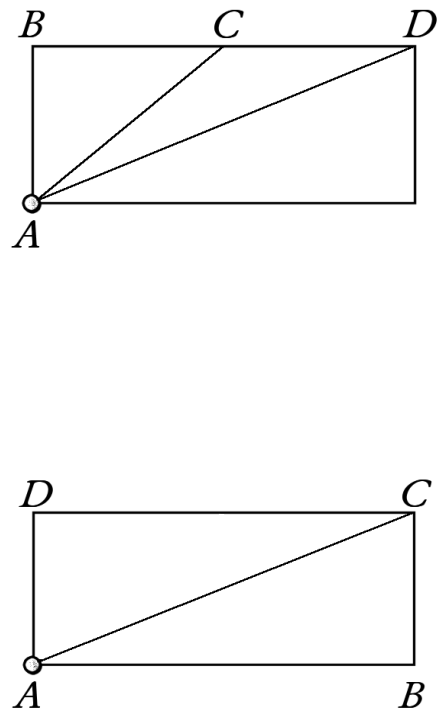

vers quelqu'endroit, est nommée determination; Il faut seulement distinguer la determination d'avec le mouvement, puisque l'un peut estre augmenté sans que l'autre change comme par exemple suposé que la bale $A$ se meuve sur la ligne $A B$ qui est perpendiculaire à la ligne $B C$ et que cette bale parcoure la distance $A B$ en une minutte, on voit que la determination de cette bale vers la ligne $B D$ sera de la quantité $A B$ en une minutte, qui est egale à son mouvement : mais si au lieu de se mouvoir sur la ligne $A B$ elle se meut sur $A C$ et quelle la parcoure aussi en une minutte, alors sa determination vers la mesme ligne $B D$ sera toujours de la mesme quantité $A B$ en une minutte, et son mouvement sera de la quantité $A C$. qui est plus grande que son premier mouvement $A B$ et enfin si la bale $A$ se meut sur la ligne $A D$ au lieu de se mouvoir sur $A B$ ny sur $A C$ et quelle la parcourre en une minutte, il est encore clair que sa determination vers la ligne $B D$ sera encore egale à la mesme quantité $A B$ et son mouvement à celle d' $A D$ qui est plus grande qu' $A B$ et qu'AC. E† que par consequent, sa determination dans ces trois mouvemens a esté toujours la mesme, quoy que les mouvemens ayent esté differends.

On peut aussy distinguer deux determinations à la fois ${ }^{16}$, dans un corps qui se meut, et l'une peut changer quoy que l'autre soit la mesme, pour concevoir cela distinctement, soit appellé $A D$ Latitude, et $A B$ Longitude, et que la figure $A B C D$, soit rectangle, et soit conceu que la bale $A$ se meuve sur la ligne $A C$ et quelle la parcoure en une minutte, il est constant que lors qu'elle sera parvenue au point $C$, elle se sera meüe $\left[6 \mathbf{v}^{\circ}\right]$ en latitude, et en longitude, et que par consequent elle aura eu une determination en latitude, et une en longitude, sçavoir celle de latitude de la quantité $A D$, et celle de longitude $A B$ mais suposant qu'on la voulust faire mouvoir une seconde fois sur la ligne $A C$ en luy donnant la mesme vitesse de mouvement que la premiere fois, et que l'air estant beaucoup plus difficile à fendre en latitude, qu'il faille une fois plus de temps à fendre l'air de ce costé là qu'auparavant sans que pour cela il soit plus dificile en Longitude que la premiere fois ; il est encore constant qu'au lieu de parcourir la ligne $A C$ elle parcourra la ligne $A F$ en une minutte, suposant que la ligne $E F$ coupe $A D$ et $B C$ en deux parties egales; parce qu'elle n'aura peû faire en latitude en une minutte, que la moitié de la première fois, c'est-à-dire $A E$ et qu'en longitude elle

qu'elle coïncide avec la tangente à la trajectoire du mobile. Descartes fait de la détermination, parce qu'elle est purement géométrique, une propriété première du mobile, contrairement à la vitesse qui découle, elle, d'un principe physique, à savoir la conservation de quantité de mouvement dans l'univers, et donc d'un principe de philosophie naturelle. Toujours par souci d'abstraction géométrique, le mouvement est essentiellement caractérisé par la trajectoire du mobile et sa détermination à chaque instant. Le temps et la vitesse sont des grandeurs en quelque sorte superposées aux deux précédentes, par l'interaction du mobile avec la matière environnante. Le raisonnement de Renau, qui reprend une démonstration du R. P. Pardies (cf. annexe $n^{\circ} 1$ ), s'inscrit donc dans une approche cartésienne de la notion de mouvement, même si, comme on le verra plus loin, Pardies a adopté quelques principes différents de ceux de Descartes. Voir P. Mouy, op. cit. à l'index des matières, « détermination ॥.

16 Cette précision revient à insister sur la différence fondamentale entre le mouvement et sa détermination. Le raisonnement et les principes qui le fondent sont exactement ceux de la Dioptrique de Descartes, Discours second. cf Descartes, CEuvres, Paris, Gallimard, 1958, p. 190. Descartes utilise l'analogie d'un joueur de jeu de paume et tient le même raisonnement : dans la rencontre avec un obstacle, on peut décomposer le mouvement du mobile en deux déterminations dont l'une concentre tout le changement intervenant dans le contact, tandis que l'autre demeure inchangée. 


\section{Mémoire sur la construction des vaisseaux}

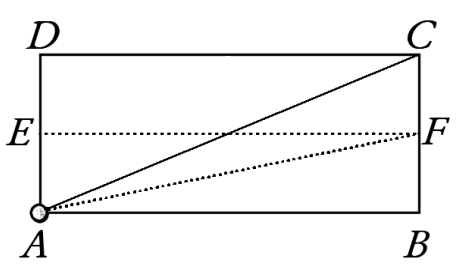

aura fait autant que la premiere fois, c'est-à-dire $A B$ et qu'il n'y a que le point $F$ qui soit eloigné de celuy d'A de la quantité d'AE en latitude, et de celle d'AB en longitude, donc elle se trouvera là au bout d'une minutte et aura parcouru AF. II ne reste plus, pour ce que j'ay besoin, que de faire veoir en quelle proportion de force ${ }^{17}$, les corps qui sont meüs, rencontrent d'autres corps qui sont en repos, et en quelle proportion ils transferent de leur mouvement à des corps qui peuvent estre meüs par leur renconstre, et sur quelles lignes ils les determinent à se mouvoir, le tout suivant les diferentes inclinations dans les quelles ils les rencontrent.

Et pour cela il n'y a qu'à considérer qu'un corps ne receoit d'impulsion par un

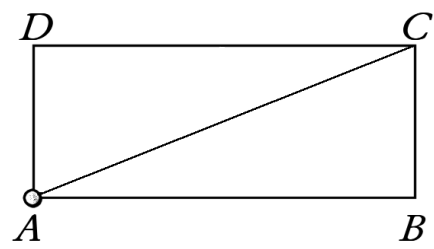
autre, qu'autant qu'il est oposé à quelque determination de son mouvement, et à proportion de la vitesse [ [ $\left.{ }^{\circ} 7\right]$ avec laquelle celuy qui se meut, rencontre l'autre, cela estant, un boulet qui iroit rencontrer deux murailles l'une apres l'autre, les forces avec lesquelles il les rencontreroit, seroient en mesme raison entr'elles que les determinations vers ses deux murailles, car soit le boulet de Canon A au lieu de la bale que j'ay dite dans la derniere proposition, et qu'il se meuve avec grande impetuosité, sur la ligne $A C$ il rencontrera $B C$ et $D C$ que je supose estre deux murailles, et comme BC n'est oposé qu'à sa determination de Longitude elle ne reçoit d'impulsion que par elle et en longitude, c'est-à-dire que si c'estoit un corps qui se meut par la rencontre de ce boulet, ce ne seroit qu'en Longitude, par consequent sur une ligne qui luy seroit perpendiculaire de mesme DC n'est aussy opposée qu'à sa determination en latitude et ne reçoit n'on plus, d'impulsion que par cette determination sur une ligne qui luy est perpendiculaire, c'est-à-dire en latitude mais si la determination en longitude est plus grande que celle en latitude la muraille $B C$ sera oposée à un plus grand mouvement que $D C$ par consequent le boulet employe plus de force contre $B C$ que contre $D C$ en mesme proportion, que $A B$ est plus grande qu' $A D$ parce que suposant que $A B$ soit double de $A D$ le boulet ne fera qu'une toise en latitude lors qu'il en fera deux en longitude, par consequent rencontrera $B C$ avec une fois plus de vitesse que DC ainsy avec une fois plus de force, si triple trois fois \&ca.

Tout cela bien entendu il n'est pas dificile de resoudre la question du vaisseau ; car soit la figure de la partie du vau qui est dans l'eau ABCDdcb, dont le cap soit $A, B b$ la vergue $\left[7 v^{\circ}\right]$ de mizaine, Cc celle du grand mat, Dd celle du mat d'artimon ou du perroquet de fougue ${ }^{18}$, et que les voilles qui y sont le long, estant amurées ${ }^{19}$ à estribord, et bordees à basbord, ne peuvent pas varier ; il est clair que le vent venant d'F rencontrera la voilure suivant la ligne FE determinera le vaisseau par son impulsion contre ces voiles, à se mouvoir sur une ligne qui leur sera perpendiculaire, c'est-à-dire sur EG comme j'ay demontre cy devant, et que faisant la figure AEOG rectangle, le mouvement du vaisseau sur la ligne

17 L'emploi du terme de « force ॥ nécessite le plus grand discernement : il ne s'agit certes pas de l'acception moderne du terme de "force " (pour lequel Descartes et ses successeurs emploient souvent le terme de " puissance "), mais plutôt de l'intensité d'une grandeur mécanique : les Cartésiens parlent toujours de force de mouvement, force de vitesse, et même force de repos (il faut une certaine quantité de mouvement pour mettre en mouvement un solide au repos), sans détacher le terme de force de son objet.

18 La fougue est une voile carrée que porte le mât établi au-dessus du mât d'artimon et qu'on nomme le mât de perroquet de fougue.

19 Amurer est fixer l'amure d'une voile selon l'angle qui lui fera recevoir le vent. L'amure est le cordage fixant le point d'en bas, nommé point d'amure, d'une basse voile qui se trouve au vent. Dans son Glossaire nautique A. Jal cite Fournier : « amurer est pezer à force d'hommes sur les couetz d'une voile pour tenir le point de la voile sur le bord, vers le vent ».

150 Documents pour l'histoire des techniques $n^{\circ} 16$ - décembre 2008 


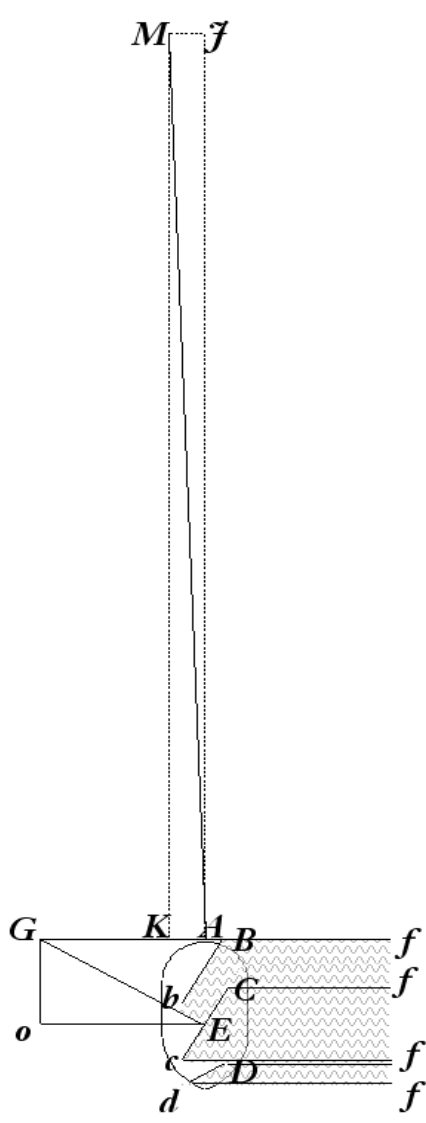

$E G$ a deux determinations, l'une en latitude et l'autre en longitude, prenant $A E$ pour la latitude, et $A G$ pour la longitude, et si le vau n'avoit pas plus de dificulté à fendre l'eau d'un costé que d'autre, il se mouveroit sur la ligne EG mais comme il est beaucoup plus long que large il y a beaucoup plus d'eau qui s'oppose à sa determination en longitude qu'à celle de latitude, et par consequent EG ne sera pas la route du vaisseau, ce sera une ligne beaucoup plus pres du vent, et pour la déterminer, il faut suposer qu'à cause qu'il y a beaucoup plus d'eau qui s'oppose par son costé que par devant, qu'il y a aussy cent fois plus de dificulté à fendre l'eau de ce costé là que de l'autre, mais que si cette dificulté n'estoit pas plus grande que par son devant, il parcoureroit la ligne EG en une heure par le mouvement que le vent luy transfereroit, sur ce pied là il feroit $A G$ en longitude, que je suppose estre de cinq quarts de lieve, et EA en latitude que je suppose aussi estre d'une demy lieve, il est constant que comme il trouve cent fois plus d'obstacle, à sa determination en longitude qu'en latitude par ma suposition ${ }^{20}$, il sera aussy cent fois plus de temps à faire cinq quarts de lieve en longitude, c'est-à-dire qu'en vingt heures [f ${ }^{\circ} \mathbf{8}$ ] il n'en fera qu'un quart de lieve, mais comme en latitude il fait une demy lieve par heure, il en fera dix en vingt heures, c'est pourquoy si je prends EJ sur EA prolongée, égale à dix lieues, et AK sur AG égale à un quart de lieve, tirant la ligne $K M$ et $J M$ paralelles à $E J$ et à $A G$ le point où ces deux lignes se couperont, c'est-à-dire $M$ sera celuy où le vaisseau se trouvera au bout de vingt heures de sa route, n'y ayant que ce point qui soit à dix lieues de latitude et à un quart de lieve de longitude du point $E$.

Comme toutes les situations de la vergue ne sont pas également avantageuses ${ }^{21}$ pour gaigner le vent à un vaisseau ${ }^{22}$, ou pour quelqu' autre chose, où on ait besoin de menager le vent ${ }^{23}$, et avancer en mesme temps, n'estant pas toujours

20 Renau reprend, sans y faire référence, l'hypothèse du Père Pardies dans son ouvrage La statique ou la science des forces mouvantes (Amsterdam Pierre de Coup 1724 (1 ère édition 1673) pp. 234-235; si le vaisseau se déplaçait avec la même facilité dans toutes les directions (ce qui serait le cas s'il avait une forme circulaire), il parcourrait en une heure 1/2 lieve en latitude et 5/4 de lieve en longitude.

Mais avec une résistance latérale cent fois plus importante, quand il parcourt 1/2 lieve par heure en latitude, il ne peut parcourir en une heure que 5/400 lieve. Renau présente le résultat différemment en examinant l'espace parcouru en 20 heures:

- en latitude, le vaisseau parcourra 1/2 lieve/heure $\times 20$ heures $=10$ lieves.

- et en longitude, le vaisseau parcourra 5/400 lieve/heure x 20 heures = 1/4 de lieve

21 Renau rompt avec ses considérations de figure du vaisseau pour aborder celles de manœuvre lorsqu'on navigue au près. Ce qui est tout à la fois répondre à la question soulevée avec Deane et Desclouzeaux en s'appuyant sur les données théoriques préliminaires et montrer que ces dernières sont également applicables à la construction et à la manœuvre.

22 Remonter dans la direction d'où vient le vent.

23 Pour remonter contre le vent il n'est pas toujours favorable d'être au près serré c'est à dire de rechercher le plus faible angle de la voile par rapport à la direction du vent apparent. En effet, le vent que reçoivent les voiles d'un vaisseau animé d'une certaine vitesse n'a ni la même direction ni la même vitesse que le vent réel. Le vent créée par la vitesse du bateau (vent relatif) se combine au vent réel pour donner le vent apparent qui agit sur les voiles, c'est aussi celui qui est ressenti sur le bateau par les instruments et par l'équipage ; le vent apparent fait toujours un angle plus aigu avec l'axe du vaisseau que le vent réel, la différence angulaire peut atteindre 30 à $35^{\circ}$, c'est pourquoi Renau évoquait des angles de plus ou moins $45^{\circ}$ angle du vent apparent avec le cap, alors que des voiliers ne faisaient rarement plus de six rhumbs $\left(6 \times 12,5=75^{\circ}\right)$ mesurés par l'angle du vent réel avec l'axe du 


\section{Mémoire sur la construction des vaisseaux}

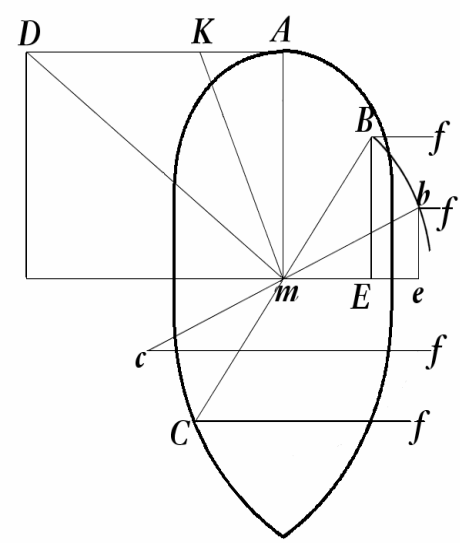

le meilleur de tenir le plus pres qu'il se peut du vent; on pourra terminer ${ }^{24}$ celle qui convient le plus, pour faire la route la plus avantageuse selon le dessein que l'on a, car à la vérité plus la vergue est inclinée contre le vent, plus le vaisseau va pres du vent, mais il receoit moins d'impulsion par le vent à proportion qu'il va plus pres du vent, et outre cette diminution de force, il y en a une autre qui vient de ce que la vergue estant plus inclinée il y a une moindre quantité de vent qui la pousse encore à mesme proportion, ce qui fait qu'il perd en vitesse en raison doublée de ce qu'il gaigne à venir au vent lors que le vent vient perpendiculairement sur la quille du vau, pour le demontrer soit les deux situations de vergue $B C$ et bc, lors qu'elle est en $B C$ elle receoit plus d'impulsion par le vent qu'en bc en mesme raison qu'en BE est plus grande que be comme j'ay desja fait veoir ailleurs, et $\left[\mathbf{8}^{\circ} \mathbf{}\right]$ la quantité de vent qui pousse la vergue en cette situation, sçavoir celle qui est entre FBCF est plus grande que celle qui la pousse en la situation bc sçavoir celle qui est entre fbcf en mesme raison que BE est plus grande que be ainsy la vergue venant de la situation $B C$ à celle de bc le vaisseau perd en vitesse en raison doublée ${ }^{25}$ de $B E$ à be et ne gaigne à venir au vent qu'en raison d'AD à $A K$ et en cas que le vent vienne perpendiculairement sur la quille, DA sera à DK comme BE à be parce qu' alors ces quatre lignes seront quatre costés semblables de quatre triangles équiangles deux à deux, sçavoir DAM. et BEM ; KAM : beM ${ }^{26}$.

vaisseau ; aux allures de "près », c'est-à-dire lorsque le vaisseau va contre le vent, le vent paraît plus fort et pourtant le bateau ralentit, car la composante axiale du vent (c'est à dire celle qui assure la propulsion du bateau) est plus faible. Plus le vent apparent fait un angle aigu par rapport à l'axe du vaisseau plus sa composante axiale est faible, alors qu'au vent arrière le vent apparent (différence entre la vitesse du vent réel et la vitesse du bateau) est plus faible que le vent réel ; le navigateur doit faire un choix entre ces options pour se placer en fonction du point où il veut se diriger avec le meilleur vent apparent en direction et en force.

Cet antagonisme (force du vent - effet sur la voilure) est une bonne illustration de la contrariété, que les experts et ingénieurs de l'époque moderne mettent en avant pour rendre raison des choix techniques qui doivent combiner au mieux des qualités mutuellement rebelles. On peut y voir l'amorce d'une réflexion sur l'optimisation en tant que mode de pensée dans l'ingénierie.

24 déterminer.

25 L'expression « en raison doublée » signifie qu'une grandeur est proportionnelle au carré d'une autre grandeur. Dans le texte : « C'est donc en raison doublée de BC à bc que le vaisseau perd en vitesse dans le cas 2 par rapport au cas 1 ॥ signifie que la vitesse du navire est proportionnelle au carré du cosinus de l'inclinaison de la voile par rapport au vent. La figure $4\left(p .8 v^{\circ}\right)$ du mémoire illustre le propos de Renau suivant lequel : « ce qui fait qu'il perd en vitesse en raison double de ce qu'il gagne au vent 1 . La variation des forces propulsives du vent sur les voiles et des forces résistantes qui s'opposent aux déplacements du vaisseau, lorsque le réglage des voiles est modifié, est comme les carrés des vitesses du vaisseau. Or le rapport des distances parcourues par le vaisseau avec deux positions de voiles est égale au rapport des vitesses du vaisseau, et si les voiles sont réglées au plus près du vent, la vitesse diminue, la force propulsive aussi et le bateau dérivera moins, puisque $A K$ est inférieur à $A D$.

26 Renau fait deux propositions successives: dans le cas 1, l'action du vent sur la voile $C B$ a une résultante $M D$ perpendiculaire à $C B$ et dans le cas 2 , une résultante $M K$ perpendiculaire à cb. L'impulsion du vent sur la voile dépend de l'inclinaison de la voile par rapport à la direction du vent. CB étant plus proche de la perpendiculaire au vent AM que cb l'impulsion du vent dans la voile sera dans une proportion de BE à be. Mais encore, la longueur de voile soumise à l'action du vent en $\mathrm{fBCf}$ et fbcf est également dans le même rapport de $B E$ à be. C'est donc en raison doublée de $B C$ à bc que le vaisseau perd en vitesse dans le 
Ce n'est pas que la figure des voiles n'estant pas aussy platte ${ }^{27}$ que je suppose icy et que le vent donnant contre les œuvres mortes particulierement aux vaisseaux qui sont en volumes et les diferents courants qui se peuvent rencontrer n'apportent quelque differances à ces calculs, mais tout cela peut estre considéré separement pour le sçavoir assez exactement.

Je demontrerois aussi de la mesme maniere, ce qui fait qu'un vaisseau derive moins avec ses huniers qu' avec ses basses voilles ${ }^{28}$, et en quelle proportion, aussi bien que beaucoup d'autres effets semblables qu'on remarque à la mer, mais mon intention n'estant que de donner une methode pour conduire les façons des vaisseaux j' ay seulement parlé de ce qui precede, pour tirer cette conclusion qui en est une suitte necessaire, que de toutes les lignes qui composent les façons des vaisseaux, celles-là sont les meilleures, qui sans changer ses proportions fondamentalles, font le moins de resistance qu'il se peut par devant [ [ ${ }^{\circ}$ '] comparee à celle des costez ${ }^{29}$; Je dis sans changer les proportions fondamentales, parce qu'en donnant par exemple moins de largeur aux vaisseaux, il en arriveroit ce que je dis là, mais on tomberoit d'ailleurs dans d'autres inconveniens plus fascheux que les avantages qu' on en pourroit tire ${ }^{30}$, il faut presentement veoir qui sont les lignes qui ont le plus ces proprietez là.

cas 2 par rapport au cas 1 . En revanche, l'impulsion sur la voile étant orientée suivant MD dans le cas 1 et suivant $M K$ dans le cas 2 , avec une quantité de vent égale, le vaisseau remonte mieux au vent dans le cas 2 que dans le cas 1, selon la proportion AD / AK. Dans toutes ces démonstrations, Renau ne tient pas compte de la conservation du mouvement, il ne distingue pas quantité de mouvement, vitesse et force.

27 Renau recourt ici à une simplification d'analyse classique à son époque. I. Pardies et plus tard J. Bernouilli l'utilisent également.

28 Si le vaisseau dérive moins avec ses huniers qu'avec ses basses voiles c'est parce qu'il est possible suivant Renau de " serrer » le vent avec les voiles hautes plus qu'avec les basses et qu'au près le vaisseau dérive moins.

29 « Un vaisseau est un tout composé d'une infinité de parties : \& il paraît comme impossible, quand on veut qu'une certaine qualité domine, qu'elle ne préjudicie aux autres "1, lit-on dans le Journal de Trévoux, t.XLVII, Slatkine reprints, 1969, p. 516. Selon le raisonnement de Renau, à la recherche d'un optimum, on considère que la surface latérale d'une carène est une surface gauche dont on doit rechercher la forme pour que la résistance de l'eau au déplacement frontal soit la plus réduite possible par rapport à la résistance latérale. Autrement dit, on privilégie la marche. Il y a ici un point central du mémoire, car par cette phrase Renau pose ce qu'il considère comme LE critère de la bonne façon ; or le choix de ce critère est discutable, et même daté historiquement. Par exemple, Bouguer adopte un critère de type : « minimiser l'amplitude des différents types d'oscillations ॥. E† on pourrait aussi (XXe siècle) chercher à optimiser la section des couples ou la solidité de la carène, etc. II n'y a pas de bon choix dans l'absolu, mais le choix d'un critère est révélateur d'une pensée, et sans doute aussi des moyens de modélisation disponibles pour exhiber un optimum. Si maintenant on se place du point de vue du système de la guerre navale où s'affrontent des flottes, obtenir une capacité de vitesse équivalente pour différents types de bâtiments est très important. En général la vitesse est privilégiée pour les vaisseaux de course.

30 Lorsqu'il précise qu'il ne s'agit pas de changer les proportions fondamentales, Renau prend la précaution de ne pas remettre en cause celles qui sont définies par les différents règlements ou ordonnances royales, à partir des modèles de vaisseaux existants. Cette dernière incise est un lieu commun des écrits techniques de l'époque moderne sur la nécessité de pondérer les avantages et les inconvénients qui en dépendent nécessairement (voir note 27). 


\section{Mémoire sur la construction des vaisseaux}

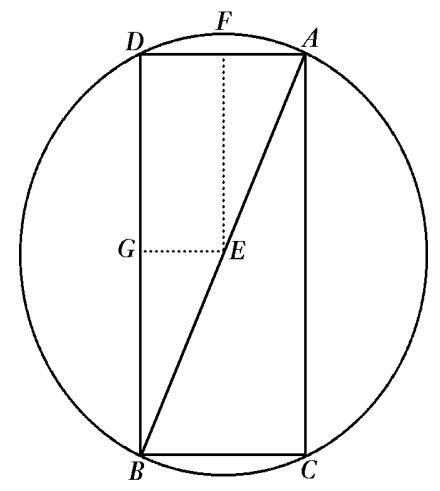

Pour ce là soit imaginé presentement que $A B C D$ qui est un quarré long, et le triangle $A B C$, soient meus de la mesme maniere que le vaisseau que je viens de parler, c'est-à-dire d'un mouvement qui ait une determination en latitude et une autre en longitude, je dis que la resistance que ce triangle reçoit par son costé est plus grande comparée à celle quelle reçoit par son devant, en raison doublée de $B D$ à $A D$ que celle que le quarré reçoit par son costé n'est à celle qu'il reçoit par son devant, en raison doublée de $B D$ à $A D$ c'est-à-dire que si le quarré long et le triangle estoient dix fois plus longs que larges, le triangle deriveroit dix fois moins que le quarré long, car l'eau qui s'oppose au costé et au devant du quarré long, s'opposant de la mesme maniere, c'est-à-dire perpendiculairement, la resistance qu'elle fait sera à celle qu'elle fait au devant en mesme raison de la quantité d'eau qui s'oppose à tous deux, c'est-à-dire comme $B D$ est à $D A^{31}$. mais au triangle, outre que la resistance qu'il reçoit par son costé est plus grande que celle qu'il reçoit par son devant à cause aussy de la plus grande quantité d'eau qui s'oppose par son costé que par son devant, en raison de $B D$ à $D A$, il y en a une autre qui vient de ce que l'eau s'oppose plus perpendiculairement par son costé que par [ $\left.\mathbf{9} \mathbf{v}^{\circ}\right]$ son devant, et je dis que cette resistance est à l'autre encore comme BD est à $D A$ cecy est un peu geometrique ${ }^{32}$; soit pour le demontrer divisé la ligne $A B$, en deux parties egales au

31 (BD/DA = 10) L'auteur du Mémoire n'imagine à aucun moment que la vitesse relative de l'eau sur un parement rectiligne de coque puisse varier (fût-ce suivant une loi très simple) d'un point à l'autre, alors même que le problème est éminemment dynamique et que l'eau possède une masse propre. Sans doute aussi n'est-il pas à l'aise pour évaluer l'effet résultant dans ce genre de situation. D'un point de vue physique, on pourrait dire que Renau n'appréhende pas l'inertie; une face plane frappée perpendiculairement oppose une réponse hydrostatique : l'inertie de l'eau et les conséquences tourbillonnaires sont négligées. En termes modernes, ce qui manque à Renau est le théorème de la quantité de mouvement d'Euler pour les fluides (donc un résultat de dynamique, applicable que la direction du jet soit perpendiculaire ou non à la coque).

32 Le raisonnement de Renau est déroutant, puisqu'il ne considère qu'un seul parement de la coque dans le cas du navire triangulaire, alors qu'il en considère deux dans le cas du navire rectangulaire. Le raisonnement s'éclaircit davantage si l'on observe que, sous les rubriques " coque rectangulaire » et " coque triangulaire », Renau examine en réalité deux angles d'attaque de l'eau sur un parement (la carène). II y a en effet, dans la forme de son raisonnement, une disjonction physique complète entre les deux situations : choc frontal, choc oblique.

Le cas du navire rectangulaire correspond, pour Renau, à la situation particulière où, en plus de la forme de cette carène, le navire marche " de bout » par rapport à l'eau, c'està-dire que le cap est perpendiculaire à la face la plus courte. Dans ce cas, le choc de l'eau sur cette face se produit perpendiculairement, ou de plein fouet, et l'effet doit être analysé, selon Descartes, comme la résultante du choc d'un très grand nombre d'atomes identiques ; autrement dit, l'effet est proportionnel à la surface battue par l'eau. Mais ce raisonnement est également appliqué sans modification à la paroi latérale par Renau : alors que d'un point de vue moderne l'effet de l'eau serait considéré comme nul (en supposant que la présence de la coque ne modifie pas le champ de courant), Renau pose que le choc ne peut être que de plein fouet sur ce parement parce que s'il existe, il n'a pas de raison d'être oblique. L'obstacle de pensée est ici comparable à celui qui empêchait les contemporains de Galilée de considérer qu'un mobile puisse avoir une vitesse instantanée nulle sans pour autant être au repos.

Notre auteur affirme que dans le cas de la forme rectangulaire, le navire s'avançant perpendiculairement au côté le plus court, la traînée (résistance frontale) est proportionnelle à la surface du petit côté, et la dérive (résistance latérale) est proportionnelle à la surface du côté le plus long, la vitesse relative de l'eau et du navire n'ayant, en intensité, qu'un 


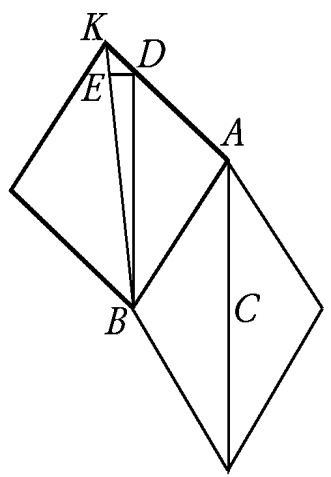

point $E$ et le prenant pour centre soit descrit un cercle de l'intervale $E A$ et soit tiré $E F$ paralelle à $D B$ et $E G$ paralelle à $D A$ : la resistance de l'eau par le costé du triangle est à celle de son devant, comme le sinus de l'angle GEB est à $F A$ de l'angle FEA, c'est-à-dire comme GB est à $F A$ comme j'ay desja demontré cy devant, et $G B$ est (à) FA comme $D B$ est à $D A$, donc la resistance au triangle par son costé, est à celle du devant en raison composée de la quantité d'eau, et de l'inclination dans laquelle elle s'oppose c'est-à-dire de deux fois BD à DA ${ }^{33}$. Je demonstrerois de mesme que toute les figures qu'on pourroit descrire dans ce quarré long, dans la partie exterieure du triangle, seroient toutes plus avantageuses que le quarré long, et moins que le triangle et que par conséquent le triangle seroit le plus avantageux s'il ne faisoit point d'angle au point $B$ avec la ligne $B D$ qui est paralelle à CA parce qu'alors il ne faudroit considérer (parlant comme j'ay fait jusqu'icy) la latitude, que le long de BA et qu'il seroit obligé de se mouvoir sur cette ligne ce qui luy feroit perdre à cause de la determination, en mesme raison ${ }^{34}$ que d'AK est plus grande comparee à $A B$ que $D E$ n'est à $D B$ ce qui seroit trop considérable comme il se verra par la seule veüe de cette figure ${ }^{35}$

rôle muet dans l'expression du rapport traînée/dérive. La résistance de l'eau à l'avancement du navire n'est pas analysée sous l'angle d'une vitesse relative de l'eau et de la carène, vitesse ayant, sur un parement de la coque, une direction et une intensité ; mais plutôt Renau considère que l'angle entre la coque et le cap est seule significative.

La forme triangulaire doit s'analyser, selon Renau, à l'aide de ce résultat fondamental trouvé dans Descartes et le père Pardies, que l'effet d'un impact sur un parement biais est proportionnel au sinus de l'inclinaison du parement. Le « navire triangulaire ॥ qu'étudie Renau est, en fait, un navire à coque rectiligne qui progresse de biais dans le courant. ॥ y a donc un " effet» de cette marche en biais sur la fraction de vitesse " efficace " pour vaincre le courant, mais en outre la vitesse résultante du navire n'est plus parallèle ni perpendiculaire à la coque, elle se résout en deux composantes non nulles : I'une selon la direction de la coque, l'autre suivant la perpendiculaire à la coque.

II est significatif que Renau considère que la pression sur une face frappée perpendiculairement par le fluide produit une résultante évidente à calculer (par un raisonnement de statique), alors que lorsque le fluide frappe la paroi de la coque avec un angle différent de $90^{\circ}$, il utilise son " théorème du sinus" (cinématique) du début.

33 Ce qui s'écrit : $R$ triangle latérale/R triangle frontale $=10 / \mathrm{sin} a=10 / \mathrm{tg} a$

Où 10 est le rapport des résistances latérales et frontales du rectangle et a l'angle d'incidence de la résistance latérale du triangle.

Donc $R \operatorname{tr}_{\text {latérale }} / R \operatorname{tr}_{\text {frontale }}=10 \times 10=100=(\mathrm{DA}: \mathrm{BA})^{2}$

alors que $\mathrm{R}$ rectangle latérale/R rectangle frontale $=10$

34 Dans le Ms. ce dernier mot est ajouté d'une autre plume.

35 On ne peut comprendre la rupture introduite dans ce passage par l'auteur avec les raisonnements précédents (la coque triangulaire serait la meilleure, mais...) qu'en considérant la conclusion vers laquelle il veut amener ses lecteurs. En praticien de la manœuvre, Renau comprend qu'une surface continûment courbe, dépourvue de points anguleux ou, selon son expression, "régulière ", contrarie moins les mouvements de l'eau qu'une surface présentant des angles vifs. Mais par cohérence avec la méthode mise en œuvre jusque là, il ne saurait pour lui être question de faire intervenir des filets fluides de vitesse différente : c'est donc encore à l'aune de son théorème du sinus que Renau s'essaye à expliciter les inconvénients, du point de vue de la manœuvre, du point anguleux (ou, comme on disait alors, parlant des couples, de "genoux») ) dans les coques polygonales. À cette fin il introduit un principe, une observation qui est sans doute le fruit de sa pratique de marin : le point anguleux « détermine » perpétuellement le navire, une fois qu'il est pris dans un courant parallèle à l'un des parements de sa coque polygonale, à conserver cette position relativement au courant. Ce principe une fois posé, Renau n'a aucune difficulté à représenter au lecteur la perte de vitesse qui résulte de cette rigidité positionnelle 


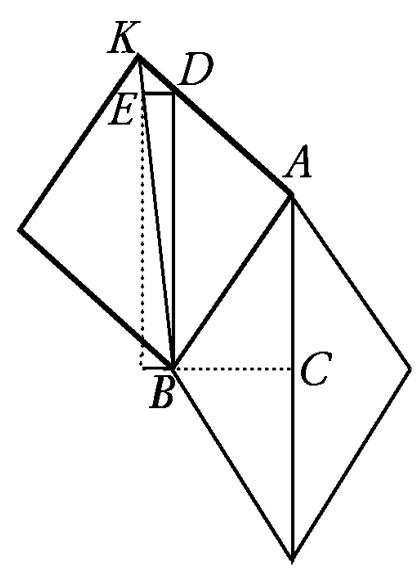

pour eviter les demonstrations qui sont trop geometriques, car le triangle estant par exemple determiné à se mouvoir sur $B K$, si la latitude estoit le long de $B D$, il seroit plus en latitude $B D$ qu'en longitude $D E$ en mesme raison que $B D$. [ $\left.{ }^{\circ}{ }^{\circ} 10\right]$ est plus grande que DE mais latitude ne pouvant estre au triangle que le long de $B A$ et la longitude le long de $A K$ il sera plus en longitude qu'en latitude, en mesme raison que $A K$ est plus grande que $B A$ et par ainsy ce que j'ay dit ; outre que le triangle se mouvant le long de $B A$ le costé $A F$ trouvera beaucoup de resistance à cause que l'eau s'y opposera assez perpendiculairement, il faut donc que la ligne qui est menee du point $B$ au point $A$ ne fasse point d'angle en $B$ ny en aucun endroit, et que par consequent soit une une ligne courbe qui ne peut estre de celles qui sont regulieres ${ }^{36}$ que cercle ou elipse parce que la parabole ny l'hyperbole ne peuvent avoir de touchante paralelle à l'axe, et d'autres raisons considerables, qui supposeraient trop de principes de geometrie pour les mettre icy, Et comme pour les proportions fondamentales des vaisseaux, on est contraint à des elipses, et ces proportions ne diferent pas tant entr'elles dans toutes les manieres de bastir qui sont en usage, qu'ils n'y satisfassent à toutes, autant que l'on peut desirer ${ }^{37}$; Toute cette methode ne concistera qu'en la description de ces lignes selon les besoins qu'on aura dans les differentes parties de construction, laquelle sera fort simple et fort commode pour ceux mesme

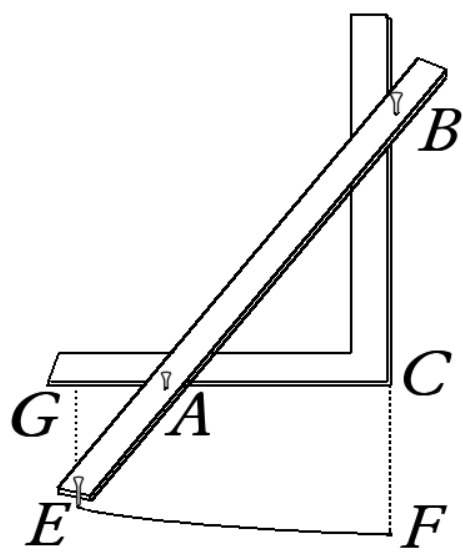
qui ont le moins d'usage dans la geometrie, par le moien de la machine qui est ci jointe ${ }^{38}$, qui ne consiste qu'en une seule équairre sur laquelle on fait mouvoir

du navire dans le courant : car, considérant toutes les faces d'une carène polygonale, certaines faces seront battue par l'eau de façon très défavorable, en sorte que la vitesse d'ensemble du navire (que Renau ne calcule d'ailleurs pas...) s'en trouvera contrariée, c'est-à-dire affaiblie.

36 C'est-à-dire parmi celles qui sont régulières.

37 Par rapport au cercle, l'ellipse offre plus de degrés de liberté : en effet, un point et la tangente en un second point déterminent complètement un cercle ; tandis qu'il faut, outre la tangente en un point, deux autres points pour déterminer complètement une ellipse. L'ellipse offre ainsi une plus grande souplesse pour produire un tracé géométrisé des façons de tous les rangs de vaisseaux, quelle que soit la méthode propre au maîtrecharpentier. En effet, si chacun avait sa « manière », pour chaque rang de vaisseaux les proportions générales variaient peu.

38 Cette « machine » n'est rien d'autre que la méthode de la bande de papier, que les Anglo-Saxons dénomment improprement " Archimedes trammel ॥ (elle n'apparaît nulle part dans les écrits d'Archimède). Selon Chasles (Aperçu historique sur l'origine et le développement des méthodes en géométrie), la méthode de la bande de papier apparaît pour la première fois dans les Commentaires sur le premier livre d'Euclide de Proclus de Lycie (410-485). À vrai dire, Proclus est très allusif : « E† quant à supposer qu'un segment se déplace dans le coin d'une équerre, son milieu décrivant un cercle, ce cercle est-il pour cela engendré de façon mixte? car les extrémités d'un segment qui se déplace ainsi, étant déplacées également, décriront une ligne droite ; et le milieu, basculant de manière égale, décrira un cercle ; mais les autres points décriront une ellipse. ") (commentaire sur la définition $n^{\circ} 4$ ) Ce passage ne constitue évidemment pas une démonstration (l'ellipse est évoquée comme une courbe intermédiaire entre la droite et le cercle), mais l'emploi par Proclus du terme d'ellipse au lieu du simple terme d'ovale porte à croire qu'il connaît une démonstration précise de ce mode de génération de l'ellipse. Au demeurant, il est tentant de rapprocher ce dispositif du générateur de conchoïde inventé par Nicomède, et décrit par Eutocius dans son commentaire du traité d'Archimède sur la Sphère et le Cylindre. Le commentaire de Proclus a peut-être inspiré Guidobaldo dal Monte, qui décrit le même instrument dans sa Planisphaerium Universalium Theorica (1579). Plus près de l'époque où écrit Renau, cette méthode de tracé est évoquée en détail dans les additions de Frans van Schooten (1615-1660) à la Geometria de Descartes intitulées Exercitationum geome-

156 Documents pour l'histoire des techniques $\mathrm{n}^{\circ} 16$ - décembre 2008 
une regle qui a deux pointes, sçavoir $A$ et $B$ en telle sorte que celle d'A coule le long de la branche $A C$ et $B$ le long de $B C$ et le bout $E$ de cette regle déscrit par son mouvement une ligne eliptique; en voicy la demonstration sur laquelle on [10 $\mathbf{v}^{\circ}$ ] peut passer sans s'y arrester parce qu'elle est tout à fait geometrique et en suppose beaucoup de principes.

\section{Démonstration \\ $\mathrm{CF}=\mathrm{AE}$ \\ $K C=E B$}

petit axe

grand axe

$E G$ est paralelle à $B C$ donc $\triangle E A G$ et $\triangle C B A$ sont équiangles, et partant EB ou KC.CG : : : : EA ou CF.AG ${ }^{39}$,
c'est pourquoy
$\square K C . \square G C:: \square E A . \square A G$,
donc
$\square K C . \square K C-\square C G:: \square E A$ ou $\square C F . \square E A-\square G A$,

c'est-à-dire $\square$ GE par ainsy le point $E$ en est un de l'elipse ${ }^{40}$.

tricárum ( $2^{\mathrm{e}}$ éd. Elzevier de 1656). Le professeur hollandais y traite la question suivante : trouver le lieu décrit par le sommet d'un triangle dont les deux autres sommets glissent le long de deux droites sécantes. Sur les sources de van Schooten, Chasles écrit ceci : « La description de l'ellipse par un point d'une droite dont les extrémités glissent sur les côtés d'un angle, était déjà connue ; Guido Ubaldi et Stevin l'avaient donnée, et elle était due aux géomètres anciens, ainsi que nous l'avons dit en parlant de Proclus. Schooten la généralisait... » op. cit., p. 98.

39 «KC.CG : : EA ou CF.AG » : il faut comprendre : «KC/CG = EA/AG ou KC/CG =CF/AG ». Par ailleurs, la notation $\square K C$ doit se comprendre comme $K C^{2}=K C \times K C$.

Tandis que Galilée, Kepler, Briggs écrivaient encore systématiquement : « a est à b, comme c est à d ", ainsi que le fait Renau plus haut dans le texte, le développement des notations algébriques fit éclore peu après 1600 diverses notations pour transcrire ces relations de proportionnalité. Leur emploi perdura jusqu'au Premier Empire en France (par exemple dans les Elémens de Statique de L. Poinsot, ou dans les Éléménts de Géométrie de Legendre) et elle était toujours vivace dans l'Entre-deux guerres dans le monde anglo-saxon. Jusqu'à Descartes, la notion de proportion, issue des travaux d'Eudoxe de Cnide, et exposée dans le livre $\vee$ des Éléments d'Euclide, joua un rôle central dans la composition des longueurs, et plus généralement des grandeurs continues. Voir sur ce sujet J. Dhombres, «La culture mathématique au temps de la formation de Desargues : le monde des coniques ॥, in " Desargues en son temps " Paris, Blanchard, 1994, pp. 55-85 ; et V. Jullien, " Le concept euclidien de proportion», La science classique, Paris, Flammarion, 1998 pp. 506-509. Le symbole : : utilisé ici par Renau, et qui a eu la plus grande longévité dans les manuels de géométrie, avait été introduit par l'anglais William Oughtred (1574-1660), I'un des inventeurs de la règle à calcul, dans son ouvrage Clavis mathematicae publié à Londres en 1632. Mais on trouve déjà chez Thomas Harriot (1560-1621) dans des manuscrits des années 1610 :

$$
\begin{array}{lllll}
1 & \prime & & \cdots & \cdots \\
a_{1} & b & : & c & d
\end{array}
$$

L'astronome anglais Vincent Wing (1619 - 1668) introduisit la notation $a: b:: c: d$ dans son livre Harmonicon celeste, tandis que Newton écrit dans les Principia de $1687 \mathrm{a}: \mathrm{b}=\mathrm{c}: \mathrm{d}$. Enfin dans l'Encyclopédie (1751) D'Alembert présente comme usuelle la notation a . b : c . d 40 Renau s'appuie sur la propriété suivante : étant donnés trois points alignés $K, C$, F et CG la perpendiculaire à $K F$ issue de $C$, tout point $E$ vérifiant la proportion :

$$
\frac{K C^{2}}{K C^{2}-C G^{2}}=\frac{C F^{2}}{G E^{2}}
$$

(où $G$ est la projection orthogonale de $E$ sur la droite $C G$ ) est situé sur une ellipse de centre $C$, dont les demi-grands axes sont CF et CK. En effet, si l'on trace le cercle de centre $C$ et de rayon $K C$, et que, prolongeant $G E$ par delà le point $E$, l'on porte le point $D$, intersection 


\section{Mémoire sur la construction des vaisseaux}

On demonstrera de mesme que tous les points où il se trouvera en seront.

Mais pour la description de ces elipses estant necessaire de sçavoir la grandeur de leurs axes dont les moitiez sont representees par la regle de cette machine sçavoir le grand par EB et le petit par EA, et que dans la conduitte des acculemens, des ouvertures de varangues, et conduite de la ligne du fort, on n'a point la connoissance des axes des elipses qui en sont necessaires, mais seulement de certaines proportions qui les terminent et qui peuvent servir à les trouver, voilà un calcul analitique pour cela dont l'aequation servira de formule pour trouver tous les axes de toutes ces elipses qui sont necessaires tant pour toutes les diferentes choses de construction que pour toutes les manieres de bastir ; sur lequel on peut passer legerement à cause qu'il est beaucoup geometrique ; mon dessein n'estant que de donner apres cela, l'explication de cette equation, qui servira universellement à trouver tous les axes qu'on aura besoin, sans plus faire aucun calcul analitique.

Voila les proportions de construction qui sont ordinairement terminées, et ausquelles on s'assujetit41 , sçavoir

de ce cercle et de la droite (GE), on aura: $K C^{2}-C G^{2}=D C^{2}-C G^{2}=D G^{2}$ (car $D$ étant sur le cercle de rayon $K C$, on a $D C=K C$ ) de sorte que la relation ci-dessus n'exprime rien d'autre que la proportion " GE est à GD comme CF est à $K C$ » : autrement dit, l'ellipse est l'image d'un cercle par affinité.

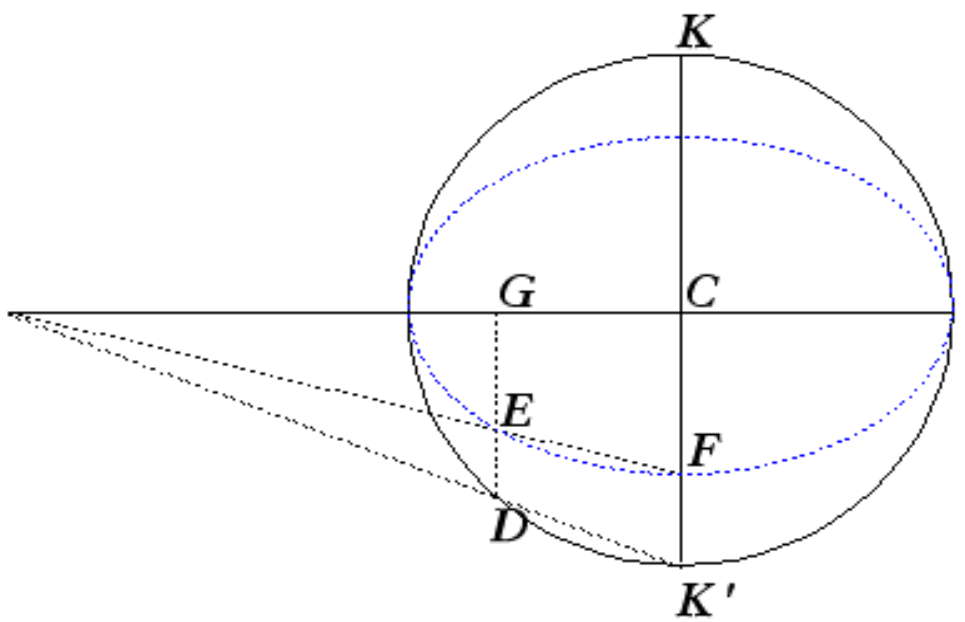

Renau utilise cette même caractérisation dans le dernier procédé de construction indiqué dans le mémoire (folio 16), lorsque sa machine est trop petite pour former les arcs les plus allongés décrivant la carène. Comme Renau en convient lui-même, cette démonstration s'adresse aux "Géomètres » : il n'est pas question pour lui de parler de l'ellipse comme étant une courbe de forme ovale, mais bien plutôt d'un lieu géométrique, c'est-à-dire d'un ensemble de points ayant en commun une propriété donnée. Seule cette caractérisation intrinsèque permet d'assigner à la courbe construite des propriétés absolves et un tracé parfaitement reproductible. En l'occurrence, Renau caractérise l'ellipse par une relation que l'on trouve également chez Archimède (Traité sur les surfaces conoïdes et sphéroïdes), et qui, faisant intervenir des rapports de longueurs (celles de cordes de l'ellipse menées d'un point du contour, à des longueurs données comme le grand axe et le petit axe), est directement exploitable graphiquement.

41 Comment décrire avec une exactitude géométrique une carène, c'est-à-dire une surface plus quelconque que les surfaces de révolution usuelles (cylindre, cône, calotte sphérique) ? Renau commence par les cotations qui caractérisent l'élévation du navire, 


\section{$c$}
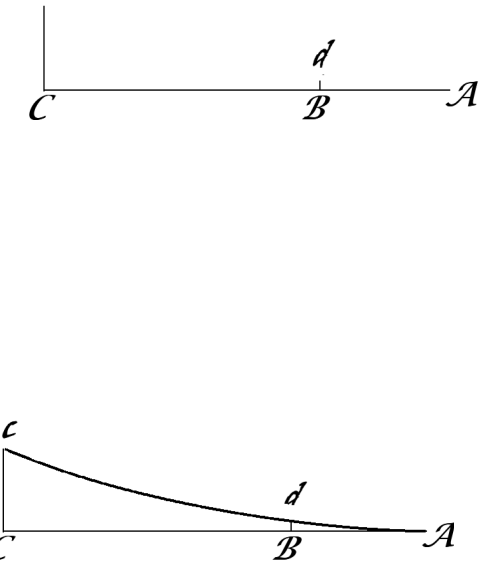

- combien il y a $\left[\mathrm{f}^{\circ} \mathbf{1} 1\right]$ du lieu de la maistresse varangue marqué dans cette figure par le point $A$. jusqu'à l'estambot $C$.

- la hauteur des façons à l'endroit de l'estambot marqué Cc

- la distance de l'endroit de la maistresse varangue jusqu'où on a accoutumé de dire que commencent les façons, marqué par $A B$

- l'acculement en cet endroit marqué par Bd,

Je ne determine point ces proportions parce qu'elles varient les unes à l'esgard des autres dans toutes les manieres de bastir, et que parlant de cette sorte ce seul exemple sufira pour toutes ces manieres à causes qu'elle(s) ne different pas tant les unes des autres, qu'une mesme nature de ligne courbe ne puisse servir dans toutes ${ }^{42}$, à terminer le reste des proportions, II faut donc presentement trouver une

c'est-à-dire la vue de tribord. Sur cette vue, il y a pour Renau deux lignes à décrire géométriquement : la ligne des acculements, et la ligne du fort. Dans la suite de l'essai, l'auteur complète cette vue par deux autres :

- le plan du navire, qui donne la variation des ouvertures de varangue (fol. $14 \mathrm{v}^{\circ}$ )

- la coupe du navire au droit du maître-couple (fol. $15 \mathrm{~V}^{\circ}$ )

Que ces trois vues, projections d'un même objet sur trois plans perpendiculaires, suffisent pour décrire la carène, cela était connu (au moins empiriquement) des techniciens du $\mathrm{XVle}$ siècle, grâce à la familiarité qui était désormais acquise avec ce " géométral ». Selon Sakarovitch, l'usage du géométral et sa pratique à l'aide de la « double projection » est apparu historiquement dans le domaine de l'architecture (Epures d'architecture, Bâle, Birkhaüser, 1997, p. 17). Ainsi, au XVIle siècle, la double projection se serait diffusée particulièrement grâce aux traités d'Abraham Bosse, de Jousse et de Derand. II faut néammoins constater que les architectes de marine anglais utilisent le géométral : c'est le cas de Baker (1580) et de Deane (deuxième moitié du 17e siècle). Ce n'est qu'en 1683 qu'une ordonnance impose en France aux maîtres charpentiers, avant la mise en construction de chaque vaisseau « d'en effectuer un modèle en carton et un profil, ou coupe perpendiculaire avec un plan, ou coupe horizontale $"$.

42 Ce passage, que Renau reprend ensuite à trois reprises ( " ... à cause qu'elles ne diffèrent pas tant les unes des autres qu'une mesme nature de ligne courbe ne puisse servir dans toutes... ॥, " comme dans toute l'Estendue des différentes proportions de vaisseaux qui sont en usage aujourd'huy il n'y a qu'une portion d'elipse qui puisse passer par ces points... ॥, « j ay desja dit que c'estoit une elipse qui satisfaisoit à toutes les proportions qui sont en usage... ॥) , éclaire bien la portée de la démarche géométrique dans le travail de standardisation. La « nature » de la ligne courbe retenue est celle de l'ellipse. Autrement dit, la seule courbe du genre conique qui permette d'interpoler entre les trois points donnés par les façons :

- la maîtresse-varangue

- l'acculement d au droit du couple de balancement arrière, à une distance a de la maîtresse varangue

- la hauteur c des façons à l'étambot, à une distance b de la maîtresse-varangue est, seIon Renau, une ellipse, dans la mesure où ces trois points respectent les « règles qui sont en usage "I, c'est-à-dire les proportions habituelles. Or il s'avère que cette condition géométrique est très restrictive : en partant d'une capacité de cale a priori, soit une longueur de quille et une ouverture au fort données, l'acculement au premier couple de balancement se voit assigner des bornes resserrées.

Si cet acculement est pris trop grand ou trop petit, la conique passant par les trois points est en général une hyperbole mais, nous dit Renau, cet acculement ne sera pas conforme dans ce cas aux proportions reçues dans l'art de conduire de façons. Le caractère très rectrictif du recours à l'ellipse (qui permet tout de même de légères modulations dans le détail) pour décrire les lignes directrices de la coque répond parfaitement à l'un des ob- 


\section{Mémoire sur la construction des vaisseaux}

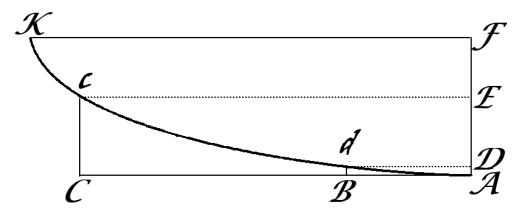

Explication des caractères

$=$ veut dire égal ainsy

$A B=a=D d$ veut dire

La ligne $A B$ egale à $a$

Est egale à Dd c'est-ò-dire

Qu'a representera la valeur de la

ligne $A B$ ou de celle

Dd qui est la mesme chose

- veut dire moins ainsy

$F E=z-C$ veut dire la ligne $F E$ egale à $z$ moins $C$ ligne courbe qui touche la ligne CA au point A pour ne point faire d'angle, et qui passe par les points $d$ et $c$, je ne diray point qu'il faut faire passer par ces points la ligne courbe la plus convenable pour la façon du vaisseau, parce qu'ils m'assujetissent à une seule ligne, n'y pouvant pas y avoir deux, pas mesme d'une mesme nature, qui y puisse passer comme je le feray veoir cy apres ; et comme dans toute l'Estendue des différentes proportions de vaisseaux qui sont en usage aujourd'huy il n'y a qu'une portion d'elipse qui puisse passer par ces points, je supposseray cet Elipse fait, dont FA est le petit axe égal à z qui est inconnu et qu'il faut trouver soit pour cela

[11 $\left.\mathbf{v}^{\circ}\right]$ Les lignes connues :

$$
\begin{aligned}
& A B=D d \\
& A C=E C \\
& B D=A D \\
& C C=A E
\end{aligned}
$$$$
\text { n................. }
$$$$
\text { (n................. }
$$

Donc FE

Par la nature de l'elipse, $\square E C$. $\square$ Dd : : $\square F A-\square F E$. $\square F A-\square F D^{43}$

c'est-ò-dire bb.aa:: $2 c z-c c .2 d z-d d$

multipliant les extremes et les moyens on a $2 a a c z-a a c c=2 b b d z-b b d d$

donc $\quad 2 b b d z-2 a a c z=b b d d-a a c c$

et enfin $z=\frac{b b d d-a a c c}{2 b b d-2 a a c} \quad$ ov $\quad z=\frac{a a c c-b b d d}{2 a a c-2 b b d}$

jectifs que s'est fixé Renau : standardiser les carènes sans aller jusqu'à figer leurs dimensions à des valeurs intangibles. En ce sens, il opère une «réduction » des méthodes en usage, à savoir le choix de l'acculement au couple de balancement, à partir des dimensions, longueur et largeur du navire, fixées par le commanditaire, par recours à un instrument (l'ellipsographe).

43 La caractérisation " archimédienne " de l'ellipse, explicitée à la note 40, est cette fois mise sous une forme où, du rapport qui existe entre les coordonnées de deux points quelconques pris sur la courbe, on déduit que la courbe est une ellipse. $B$ désignant le demi grand axe de l'ellipse, si les deux points de coordonnées cartésiennes ( $\left.x^{\prime}, y^{\prime}\right)$ et ( $\left.x^{\prime \prime}, y^{\prime \prime}\right)$ appartiennent à l'ellipse, alors : $x^{\prime} / x^{\prime \prime}=\left(B^{2}-y^{\prime 2}\right) /\left(B^{2}-y^{\prime \prime 2}\right)$, les longueurs $y^{\prime}$ et $y^{\prime \prime}$ étant mesurées le long du demi grand axe de longueur $B$, et les longueurs $x^{\prime}$ et $x^{\prime \prime}$ "le long de la droite qui lui est perpendiculaire, et depuis le point d'intersection des deux droites. Si deux points vérifient cette relation, alors on peut dire qu'ils sont sur une ellipse dont l'un des deux axes principaux a une longueur $B$, mais on ne peut dire quelle ellipse : il y en a une infinité, la longueur de l'autre axe ne pouvant être précisée sans faire intervenir un troisième point... Mais il suffit à Renau de caractériser la courbe comme étant une ellipse.

La relation entre deux points appartenant à une même ellipse dont le grand axe est donné, que Renau utilise ici, peut se déduire de la propriété commentée ci-dessus (note 40). II s'agit, là encore, d'une relation entre les rapports des diamètres menés depuis ces deux points. Quoique cette nouvelle caractérisation soit de portée moins générale que la précédente (il y a une infinité d'ellipses passant par deux points, et de grand axe donné), elle suffit parfaitement au propos de l'auteur du présent mémoire. 
Si cette equation est positive la ligne courbe qui passe par ces points est une portion d'elipse qui aura pour petit axe l'une ou l'autre de ces deux equations.

Et pour avoir le grand axe, je l'appelle $y$; et parce que par la nature de l'elipse, $\square$ GF. $\square$ Ec : : $\square A F . \square A F-\square F E^{44}$

c'est-à-dire,

$$
\begin{aligned}
& \text { yy.bb: : } \frac{b^{4} d^{4}-2 b b d d a a c c+a^{4} c^{4}}{4 b^{4} d d-8 b b a a c d+4 a^{4} c c} \cdot \frac{b b c d d-b b c c d}{b b d-a a c} \\
& \text { En divisant } \frac{b b c d d-b b c c d}{b b d-a a c} \text { parbb } \\
& \text { on aura yy. } 1: \frac{b^{4} d^{4}-2 b b d d a a c c+}{4 b^{4} d d-8 b b a a c d+4 a^{4} c c} \cdot \frac{c d d-c c d}{b b d-a a c} \\
& \qquad y y=\frac{b^{4} d^{4}-2 b b d d a a c c+a^{4} c^{4}}{4 b b c d^{3}-4 b b c c d d-4 a^{4} c c+4 a a c^{3} d}
\end{aligned}
$$

II suit de la premiere equation que si $2 b b d$ - 2aac est egal à 0 , la ligne courbe qu'on pourra mener par les points $d, a, e$, sera une parabole, car alors

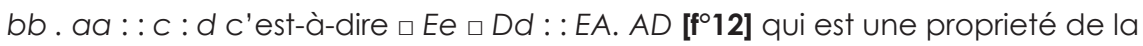
parabole.

Que si l'exposant de $\frac{b b d d-a a c c}{2 b b d-2 a a c}$ est negative ce sera une hyperbole,

Si bbdd - aacc est egal à 0 , ce sera une ligne droite parce qu' alors

$b b . a a:: d d . c c$ ou b. $a:: d . c$, c'est-à-dire Ee. Dd : :EA . AD qui est une propriete d'un triangle rectiligne.

E† enfin si lors que c'est un elipse les deux axes sont egaux, ce sera un cercle.

Ces proportions pourroient donc varier de telle maniere que ce seroit tantost un elipse, un cercle, une parabole, une hyperbole, et tantost une ligne droite ; mais comme jay desja dit que c'estoit une elipse qui satisfaisoit à toutes les proportions qui sont en usage, il faut donner icy l'explication de cette equation qui en donne la grandeur des axes, apres quoy la description n'en sera pas dificile.

Les lettres, $a, b, c, d$, representent des quantitez sçavoir $a$, la valeur de AB c'està-dire la distance qu'il y a depuis la maistresse varangue jusqu'où il est dit que commencent les façons ; $b$, represente la valeur de AC, qui est la distance de la maistresse varangue jusqu'à l'estambot, $c$, la valeur de Cc qui est la hauteur des façons à l'endroit de l'estambot; $d$, celle de l'endroit où commencent les façons, sçavoir $B D$;

44 C'est encore la caractérisation " archimédienne " ou " euclidienne " de l'ellipse, qui a été explicitée à la note 40. Le point $G$ est défini comme l'extrémité du grand axe. C'est un point de construction (donc un point abstrait), qui est en général en dehors de l'emprise de la carène. 


\section{Mémoire sur la construction des vaisseaux}

Presentement, lors que deux lettres se trouvent jointes ensemble comme ab, cela représente la valeur d'a multipliée par celle de b, ainsy ab represente le produit de la multiplication de la valeur d'a multipliée par celle de b, lors qu'une mesme lettre se trouve deux fois comme, aa, cela signifie le quarré d'a, c'est-àdire le produit de la valeur d'a multipliée par elle-mesme, et lors que deux lettres se trouvent chacune deux fois, comme bbdd cela veut dire la valeur du quarré de $b\left[12 v^{\circ}\right]$, multipliée par celle du quarré de $d$, mais si l'une se trouve deux fois et l'autre rien qu'une fois comme bbd c'est-à-dire le quarré de b, multiplié par la simple valeur de $d$, ainsy du reste.

Eł lorsque deux lettres sont séparées par une croix comme $a+b$, c'est-à-dire la valeur d'a adjoutée à celle de b, et bbdd + aacc c'est-à-dire la valeur de bbdd comme j'ay expliqué cy-devant, adjoutée à celle de aacc, mais si deux lettres sont séparées par une ligne tracée de travers comme b-a c'est-à-dire la valeur de a soustrait de celle de b, ainsy bbdd-aacc signifie la valeur de bbdd moins celle d'aacc, c'est-ò-dire celle d'aacc, soustrait de celle de bbdd,

Et enfin lorsqu'une lettre est au dessus d'une autre séparée par une ligne comme b/a c'est-à-dire la valeur de b divisée par celle d'a ainsi représente le produit de la division de la valeur de b par celle d'a

c'est pourquoy $\frac{b b d d-a a c c}{2 b b d-2 a a c}$ représente le produit de la division de

la valeur de, aacc - bbdd par celle d'a, et pour éclaircir l'obscurité qui pourroit encore y rester je vays mettre la valeur de cette equation en nombres.

Supposé donc qu'il y ait depuis la maistresse varangue jusqu'à l'estambot marqué par, b,

80 : pieds

jusqu'où commencent les façons par, $a$,

27 : pieds

la hauteur des façons à l'estambot par, c,

$14:$ pieds

l'acculement où commencent les façons par, $d$,

15 : pouces

sans comprendre dans ces deux derniers articles quelques pouces d'acculement à la maistresse varangue, qu'on donne ordinairement aux vaisseaux de cette grandeur ainsy les façons monteront à 14 pieds 9 pouces et où commencent les façons il y aura 2 pieds d'acculement [ [ $\left.{ }^{\circ} 13\right]$. 
Ainsy donc la valeur d'a estant

27. pieds, celle de c,

14.

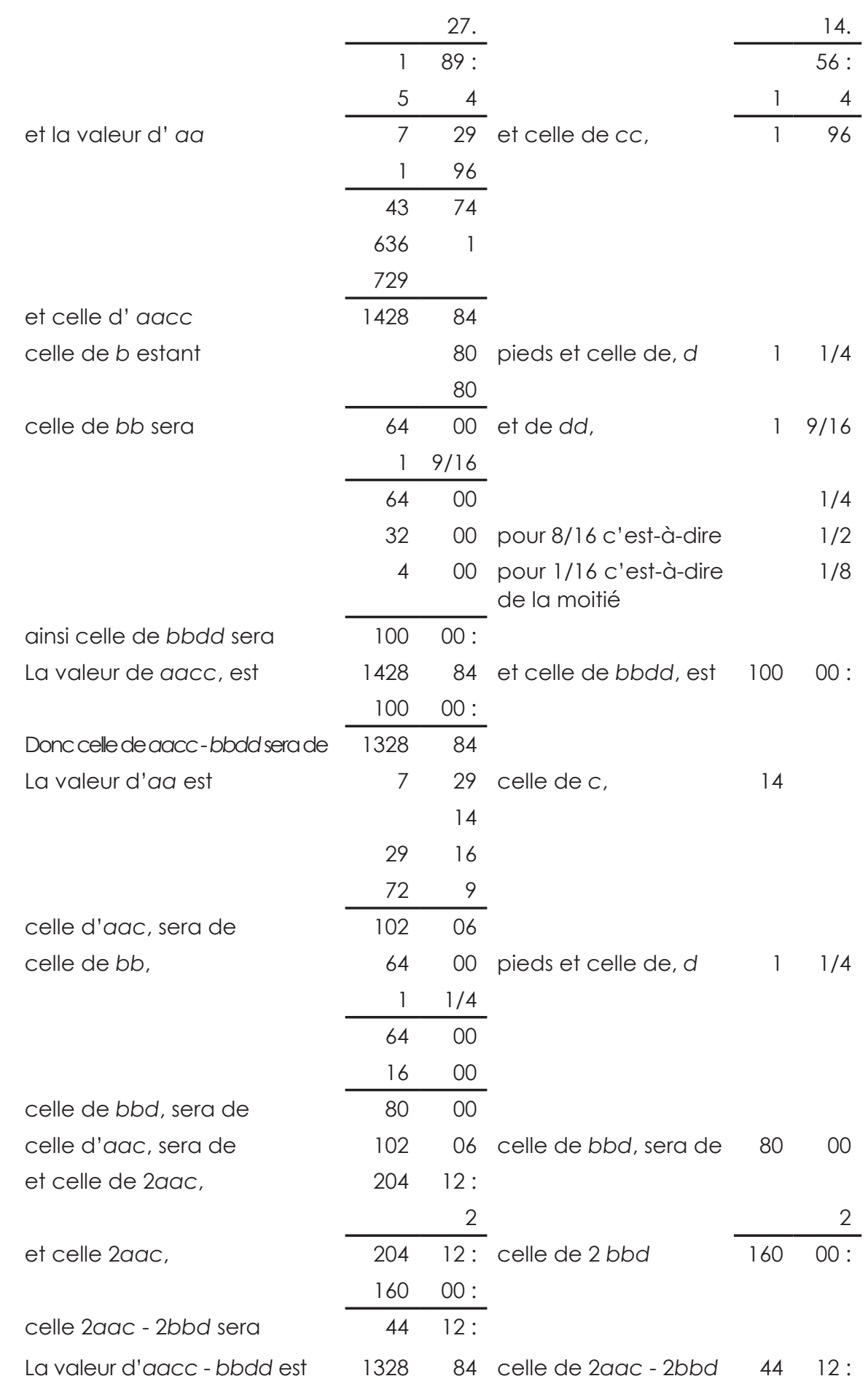




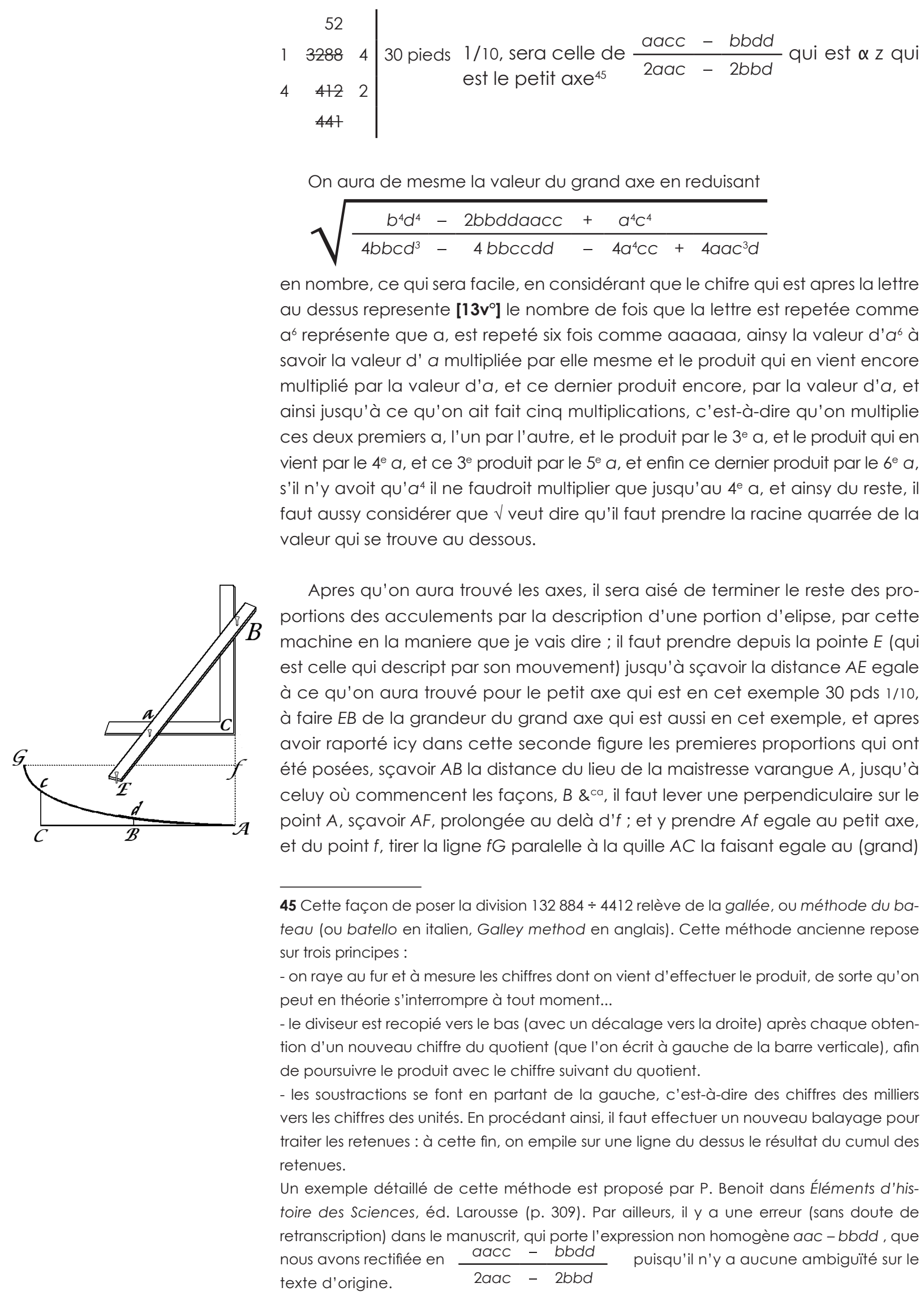

164 Documents pour l'histoire des techniques $\mathrm{n}^{\circ} 16$ - décembre 2008 

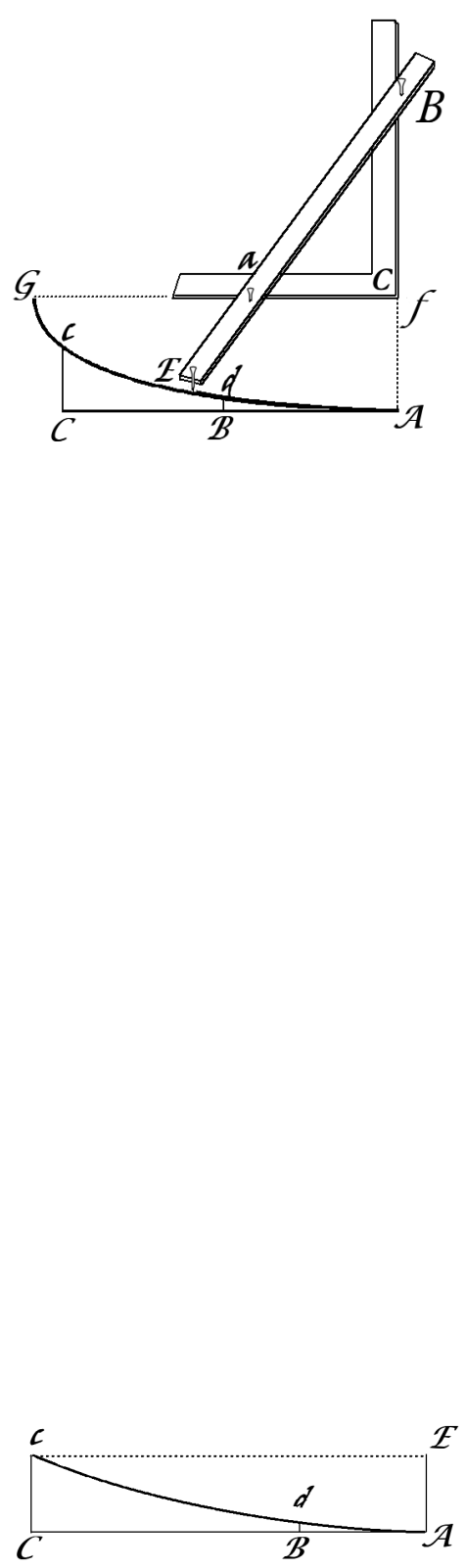

axe, ensuite de cela il faut mettre la branche $a C D$ de l'équairre, le long de la ligne $f G$. et la branche $C B$ le long d'Af prolongée comme on le voit dans cette derniere figure et faire mouvoir la regle $E a B$, en telle [ [ $\left.{ }^{\circ} 14\right]$ sorte que la pointe $a$, coule le long de la branche $a C$, et aille de $B$, le long de $B C$; la pointe $E$, par son mouvement passera en $d$, et en $c$, et descrira la portion d'elipse $c d A$, laquelle terminera tous les acculements depuis la maistresse varangue jusqu'à l'estambot cecy n'a pas besoin de demonstration, estant trop evident par celle que j'ay faite pour faire veoir que cette machine descript des elipses, et par le retour du calcul analitique.

Il faut remarquer icy que cette equation $\frac{a a c c-b b d d}{2 a a c-2 b b d}$

representera toujours la grandeur du petit axe qu'on aura besoin pour descrire les elipses qui sont necessaires pour terminer tous les acculements de tous les vaisseaux de toutes les manieres de bastir, en faisant toujours qu'a represente la distance de la maistresse varangue jusqu'où commencent les façons ; b celle jusqu'à l'estambot ; c la hauteur des façons en cet endroit, et $d$, l'acculement de l'endroit où commencent les façons, car lors que ces distances, ces hauteurs et ces acculements changeront, il n'arrivera autre chose que des changements de valeur à ces lettres, semblables à ceux qui arrivent aux grandeurs quelles representent ; mais il n'y en aura point dans la maniere d'opperer puisque ces lettres n'especifient pas plustost une grandeur qu'une autre ; Ainsy ce que je viens de faire pour les acculements de derrière, doit servir d'exemple pour ceux de l'avant, car il n'y a qu'à faire servir ces dernieres figures en faisant que AC soit la distance de la maistresse varangue jusqu'à l'estrave de l'endroit où finissent les façons ; $A B$ la distance depuis la maistresse varangue jusqu' où commencent les façons; CC, la hauteur des façons à l'estrave ; $B d$, l'acculement de l'endroit où commencent les façons en avant; et donnant aux lettres $a, b, c, d$, la valeur [14 $\left.v^{\circ}\right]$ de ces grandeurs qu'elles representent comme aux acculements de deriere ; aacc - bbdd donnera la grandeur du petit axe, et avec celle du grand; $2 a a c-2 b b d$ et on fera le reste comme j'ay dit dans l'autre exemple.

La mesme figure et le mesme exemple servira d'instruction pour les ouvertures ${ }^{46}$ c'est-à-dire la longueur des varangues et des fourcats à l'endroit des acculements ; car tirant $C E$ paralelle à la quille $A C, A C$ sera toujours la distance de la maistresse varangue à l'estambot pour l'arriere, et à l'estrave pour l'avant, $A B$ jusqu' où commencent les façons de derriere lors qu'on fera pour derriere ; et pour devant quand ce sera pour devant CC ; ou EA, qui est la mesme chose, la moitié de la longueur de la maistresse varangue ; $B d$, la diminution de la varangue à l'endroit où commencent les façons, et en donnant aux lettres $a, b$, $c, d$, la valeur de ces grandeurs, sçavoir de celles de derriere lors qu'on fait pour derriere ; et de celles de devant quand on fait pour devant car les grandeurs y sont diferentes; on trouvera les axes de l'elipse qui est necessaire pour terminer, par sa description, ces ouvertures laquelle se fera par la machine, de la maniere que je viens de l'expliquer ;

On conduira aussi la ligne du fort, depuis le lieu du premier gabarit c'est à dire du lieu de la maistresse varangue, en avant et en arriere de la mesme maniere

46 L'auteur en vient maintenant à une autre vue du géométral : le plan du navire ou « coupe horizontale "), qui doit mettre en évidence les ouvertures des varangues et du fort, depuis l'étambot jusqu'à l'étrave. 


\section{Mémoire sur la construction des vaisseaux}
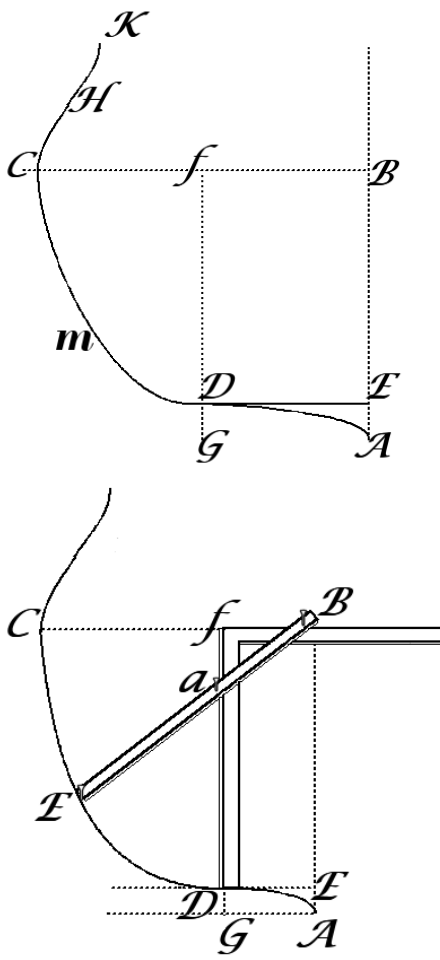

que ce qui precede, car prenant le point $A$ pour le lieu du plus large, et tirant $A C$ paralelle à la quille, AC sera distance qu'il y a depuis ce gabarit jusqu' aux Estains c'est à dire au gabarit de l'arcasse ; le point, c, celuy jusqu'où monte le fort en cet endroit, ainst, Cc, de combien le fort est plus elevé à l'endroit de l'estambot qu'au premier gabarit ; le point, $d$, celuy jusqu' où monte le fort au gabarit de [f'15] l'endroit où commencent les façons; et donnant aux lettres $a, b, c, d$, la valeur de ces grandeurs que je viens de nommer; on aura les axes de l'elipse qui sera necessaire pour terminer, par sa description, cette ligne du fort laquelle se fera encore de la maniere que j'ay dite, on trouvera de mesme la ligne du fort en avant et n'y ayant aucune diference que dans les mesures, ce qui n'apporte aucun changement dans l'opperation, je n'en diray pas davantage ;

II n'est pas necessaire non plus de donner aucun exemple, pour terminer la largeur du vaisseau le long de la ligne de son fort, ce que j'ay dit touchant L'ouverture des varangues en pouvant suffizamment servir.

II ne reste plus qu'à parler de la maniere de former tous les gabarits ${ }^{47}$, qui est ce qu'il y a eu jusqu'icy de plus dificile dans la construction, et ce qui sera le plus aisé et le plus simple dans cette methode, un seul exemple pouvant servir d'instruction pour tous, n'y ayant aucune diference dans la maniere ; Je l'expliqueray ainsy.

Il faut prendre la hauteur de la ligne du fort, la largeur du vaisseau à la ligne du fort, l'acculement de la varangue ou du fourcat et la moitié de l'ouverture de la varangue ou du fourcat, tout cela à l'endroit du lieu par où on veut faire le gabarit, soit pour cela tiré la ligne $A G$, et $A B$ [ $A D$ sur le manuscrit ] perpendiculaire à $A G$ et soit pris sur $A B$, la partie $A E$, egale à l'acculement de la varangue, ou du fourcat à l'endroit par où on veut faire le gabarit, et $E D$ egale à la moitié de l'ouverture de la varangue ou du fourcat, $A B$, egale à la hauteur de la quille, jusqu'à la ligne du fort, $B C$, egale à la largeur [15 $\left.\mathbf{v}^{\circ}\right]$ du vaisseau à la ligne du fort, le tout trouvé de la maniere que l'ay dit cy devant; et il faut que $B C$, et $E D$, soient perpendiculaires à la ligne $A B$, et soit elevé $D F$, paralelle à $A B$, coupant $B C$ au point $F$, si $F C$, est plus petit que $F D$ faisant le petit axe de la machine, c'està-dire $E A$ egal $F C$, et le grand axe $E B$ egal à $F D$ de cette figure, et mettant une branche de l'equairre le long de FD, et lautre le long de FB comme on le voit par cette figure, et faisant mouvoir les deux pointes de la regle $A$ et $B$ le long de ces branches l'autre point $E$, de la regle passera par le point $C$, et le point $D$, de cette figure, et descrira par son mouvement le costé du vaisseau ou gabarit, on descrira de mesme tous les autres gabarit ; cet exemple estant sufisant pour cela je n'en parleray pas davantage, et les reverts DB et HK estant des parties de cet elipse, sçavoir DB, la mesme chose que DM, et HK que KC, il n'y a non plus aucune dificulté là dessus.

La machine qui sera necessaire pour descrire les costez des plus grands vaisseaux, ne consistera qu'en une equairre de quatre ou cinq pieds, et une regle de quinze ou seize, que deux hommes avec celuy qui fait l'ouvrage pourront manier et s'en servir avec beaucoup de Justesse et de Facilité. Mais pour ce qui est de celle qu'il faudroit pour terminer les acculements, les ouvertures, la ligne

47 La coupe au droit du maître-couple forme la troisième vue géométrale, qui complète la description de la carène : Renau parachève ainsi la description géométrique de cette surface régulière, mais composite. 


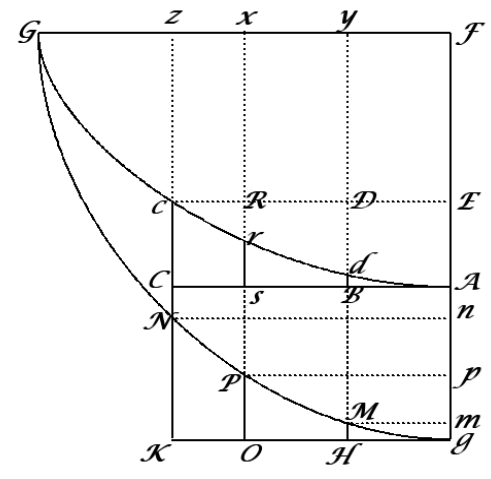

du fort, et autres choses semblables des grands vaisseaux, ne pouvant avoir une regle moins de 80 ou 90 pieds de long ; on seroit contraint de se servir d'une qui n'eust que la huitiesme ou dixiesme partie, et multiplier les grandeurs trouvées huit ou dix fois si on ne vouloit point les trouver par les calculs, dont voicy [ $\left.{ }^{\circ}{ }^{\circ} 16\right]$ une exemple qui servira pour tous.

Supposant toujours que AC soit la distance de la maistresse varangue jusqu'à l'estambot Cc, la hauteur des façons \& ${ }^{c a}$., cecy ne sera pas pour trouver par calcul les acculements qui sont entre A et $C$, sçavoir Bd, sr, CC, et les autres; ou bien les ouvertures des varangues et des fourcats, sçavoir à $A E, D d, r R$, \& ca.

Soit pour cela descrit de L'intervalle FG qui est le grand axe prenant $F$ pour centre, le quart de cercle GNPMg, et soit prolongé les lignes $z C, x s, y B$, et $F A$, et des points, $N, P, M, g$ (par où ces lignes coupent le quart de cercle) tiré les lignes $N n$, $\mathrm{Pp}, \mathrm{Mm}, \mathrm{gK}$ paralelles à $\mathrm{CA}$.

Par la nature de L'elipse et du cercle comme le grand axe FG, est au petit axe $F A$, ainsi $K N$ est à $C c, O P$ à sr, et $H M$ à $B d$, c'est pourquoy sachant les grandeurs de $K N, O P, H M$, on a par regle de trois, $C c, s r$, et $B d$, ou par une seule division qui seroit trop longue à expliquer icy; et on aura $K N, O P$, et le reste, en soustraiant $N z, P x, M y d u$ grand axe, FG, car en ostant Nz, ou Fn, qui luy est egal de FG, on aura ng, qui est egal à $N K$, ainsy du reste, et pour avoir $N z, P x, M y$ \& ca il faut considérer le grand axe comme sinus total, et $\mathrm{Nn}$, qui est egal à $\mathrm{Kg}, \mathrm{Pp}$ à og, $\mathrm{Mm}$ à $\mathrm{Hg}$, comme sinus des arcs Mg ${ }^{48}, \mathrm{Pg}$, et $\mathrm{Ng}$, et leurs complemens donneront $\mathrm{Nz}$, $\mathrm{Px}$, et $\mathrm{My}$, car $\mathrm{Nz}$ est le sinus complement du sinus $\mathrm{Nn}$ et par consequent de $\mathrm{Kg}$, de mesme $P x$, est complement ${ }^{49}$ de $P p$, et ainsy du reste.

C'est pourquoy voulant avoir par exemple l'acculement de la [16 $\mathbf{v}^{\circ}$ ] varangue ou du fourcat de l'endroit, s, il faudra diviser le grand axe en cent parties, chacune desquelles vaudra mille, parce que le sinus total, dans les livres, est de cent mille ; et regarder combien il y a de ces cent parties depuis, A, jusqu'à, s, ou de o à $g$ qui est la mesme chose ; et prenant autant de mille qu'il y a de parties, il faut chercher dans les colonnes des sinus le complement de ce nombre là, lequel osté du sinus total on aura oP ; et faisant la grandeur oP à une autre comme le grand axe est au petit cet autre sera st ${ }^{50}$, qui sera l'acculement de cet endroit ; et pour ce qui est des ouvertures on les aura en soustrayant, $C c, r t, B d$, d'AE qui est la moitié de la maistresse varangue car en ostant $r s$, de $A E$, il restera $R r$, qui est l'ouverture de cet endroit, cecy paroistra un peu long et dificile à ceux qui ne sont point versez dans les calculs de sinus; mais outre que ces calculs sont fort aisez à aprendre, il n'y a qu'à faire une fois le calcul pour un vaisseau, et on ne sera plus obligé d'en faire pour quelqu'autre que ce soit, pourvu qu'il n'y ait point de differance dans la maniere de bastir, ainsy faisant une petite table pour chaque maniere de bastir, on reduiroit aisement le reste par regle de proportion, en calculant, ou avec un compas de proportion avec lequel on pourra aussy trouver le tout sans estre obligé de se servir des sinus; On voit par cecy qu'il est aisé de répondre à toutes les questions qu'on a faites aux charpentiers par le projet de reglement; touchant les proportions des vaisseaux et d'en terminer toutes

48 «Kg » sur le manuscrit.

49 sinus des « $\operatorname{arcs} \mathrm{Mg}$ » et non des « axes $\mathrm{Kg} »$ : erreur du copiste vérifiable sur la figure.

$\mathbf{5 0}$ «sr » et non «st » : erreur encore du copiste sur le manuscrit, corrigée dans la suite de la présente transcription. 


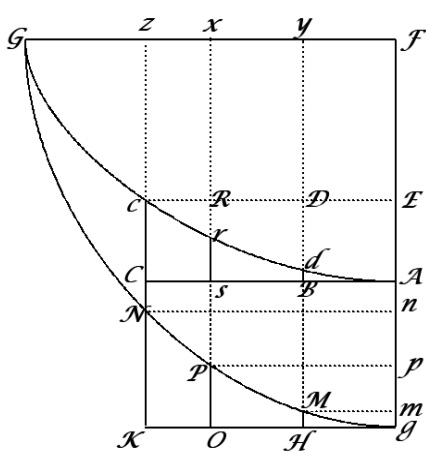

les parties, en disant la grandeur de chaque piece separement, ou apres avoir dit les principales proportions comme le lieu [ $\mathbf{f}^{\circ}$ 17] de la Maistresse varangue, la hauteur des façons \& ${ }^{\text {ca }}$ dire pour le reste comme par exemple aux acculements, toutes les varangues et fourcats auront les acculements qui seront donnez par un elipse qui a tant pour son grand axe, et tant pour son petit axe, ce qui seroit le mesme effet, que si on disoit le premier a tant, le second tant \& ca ayant terminé les deux axes d'un elipse, le reste l'estant aussy par là.

Je ne croy pas qu'il soit necessaire de faire veoir icy ${ }^{51}$ combien il en coutera moins, en suivant cette methode pour des journées d'ouvriers, puis qu'on ne sera jamais obligé de retoucher à une piece de bois, estant seur qu'elle sera bien dès la premiere fois, et qu'ordinairement il faut monter et demonter plus de quatre ou cinq fois toutes les pieces qu'on met depuis où commencent les façons jusqu'à l'estambot, desquelles on en met souvent hors d'estat de pouvoir servir coupant le fil du bois ne l'ayant pas prevu auparavant, et de quel secours elle peut estre dans une occasion de diligence, pouvant travailler indifferamment par son moyen à toutes les pieces à la fois, sans estre obligé de les mettre en place, mais ce qu'il y a encore de plus considerable c'est l'avantage qu'on peut tirer, de ce qu'on pourra retourner tant qu'on voudra à faire des vaisseaux semblables à ceux ausquels on aura heureusement reussi, et qu'on parviendra par là à des connoissances plus parfaites que celles que l'on a pour les principales proportions des vaisseaux, ce n'est pas qu'on ne soit obligé de dire à la louange des charpentiers qu'il y a à s'estonner comme ils peuvent faire de si beaux et de si bons vaisseaux, avec si peu de règle qu'ils ont ; mais s'ils ont pû juger par une longue pratique, et un bon sens des perfections et des deffauts des vaisseaux, il n'y a point de doute que ce bon sens et cette longue experience sont aidez par une methode et des regles de mechaniques, ils en pourront juger avec incomparablement plus d'assurance et de solidité qu'ils n'ont fait jusqu'icy et ne se porteront à aucun changement sans considérer autant qu'ils en seront capables tous les Inconvenients qui en pourront arriver.

51 Dans cette conclusion, Renau rassemble les arguments en faveur de la méthode qu'il préconise sous deux grandes rubriques : la première a trait à la gestion des chantiers ef porte sur l'économie en coûts et en temps de production; la deuxième grande rubrique, " plus considérable », dit-il, est que sa méthode rende possible un progrès de la marine, par une estimation plus précise des rapports entre les caractéristiques des navires et leurs qualités nautiques. La remarque ultime, sur les charpentiers est intéressante en ce qu'elle résume bien l'état d'esprit dans lequel est conçue la méthode : une manière d'éclairer le jugement des praticiens sur leurs pratiques. On est très loin de l'idée d'une technique constructive entendue comme une application de la physique mécanique. 